\title{
Catalogue of representative meteor spectra ${ }^{\star}$
}

\author{
V. Vojáček ${ }^{1,2}$, J. Borovička ${ }^{1}$, P. Koten ${ }^{1}$, P. Spurný ${ }^{1}$, and R. Štork ${ }^{1}$ \\ 1 Astronomical Institute of Academy of Scieneces, Fričova 298, 25165 Ondřejov Observatory, Czech Republic \\ e-mail: vojacek@asu.cas.cz \\ 2 Institute of Astronomy, Charles University in Prague, Faculty of Mathematics and Physics, V Holešovičkách 2, 18000 Prague 8, \\ Czech Republic
}

Received 24 September 2014 / Accepted 6 May 2015

\begin{abstract}
Aims. We present a library of low-resolution meteor spectra that includes sporadic meteors, members of minor meteor showers, and major meteor showers. These meteors are in the magnitude range from +2 to -3 , corresponding to meteoroid sizes from $1 \mathrm{~mm}$ to $10 \mathrm{~mm}$.

Methods. Parallel double-station video observations allowed us to compute heliocentric orbits for all meteors. Most observations were performed during the periods of activity of major meteor showers in the years between 2006 and 2012. Spectra are classified according to relative intensities of the low-temperature emission lines of $\mathrm{Mg}, \mathrm{Na}$, and $\mathrm{Fe}$.

Results. Shower meteors were found to be of normal composition, except for Southern $\delta$ Aquariids and some members of the Geminid shower, neither of which have $\mathrm{Na}$ in the meteor spectra. Variations in $\mathrm{Na}$ content are typical for the Geminid shower. Three populations of Na-free mereoroids were identified. The first population are iron meteorites, which have an asteroidal-chondritic origin, but one meteoroid with low perihelion $(0.11 \mathrm{AU})$ was found among the iron meteorites. The second population were Sun-approaching meteoroids in which sodium is depleted by thermal desorption. The third population were Na-free meteoroids of cometary origin. Long exposure to cosmic rays on the surface of comets in the Oort cloud and disintegration of this crust might be the origin of this population of meteoroids.
\end{abstract}

Key words. meteorites, meteors, meteoroids - catalogs

\section{Introduction}

Complex observations of individual meteoroids allow us a better understanding of the relation between meteoroids and their parent bodies. During the short luminous phase when they are called meteors, which is when a meteoroid enters the Earth's atmosphere, we have the opportunity to measure the meteoroid velocity, trajectory, and orbit with high precision. In addition, spectroscopic observations of meteors reveal the chemical composition of cometary and asteroidal meteoroids.

The study of meteor spectra started in the 1860 s with A. S. Hershel's visual observations (Millman 1963). Since the end of the nineteenth century, photographic techniques were used to observe meteor spectra (Millman 1980). Except for the systematic work by Peter Millman since the 1930s (Tors \& Orchiston 2009), there was not much general activity in this field of astronomical photography before World War II. From the 1950s onward, transmission gratings largely replaced prisms in meteor spectrographs. Extensive spectroscopic programs were carried out in the USA, Canada, the former USSR, and former Czechoslovakia (Ceplecha et al. 1998). Hemenway et al. (1971) used a sensitive video technique to observe meteor spectra for the first time. Different TV techniques and the TV data reduction methods were described by Millman \& Clifton (1975), Mukhamednazarov \& Maltseva (1989), Borovička \& Boček (1995), and Zender et al. (2004). Meteor spectra mostly consist of atomic emission lines, accompanied with some

* Spectra (Figs. 17-30) are only, Tables 4-6 are also available at the CDS via anonymous ftp to cdsarc.u-strasbg.fr (130.79.128.5) or via

http://cdsarc.u-strasbg.fr/viz-bin/qcat?]/A+A/580/A67 molecular bands and continuous radiation. Halliday (1961), Ceplecha (1971), and Borovička (1994a) provided extensive lists of line identifications in high-dispersion photographic spectra. Borovička (1994b) revealed that lines can be divided into two components - the low- and the high-temperature component.

Despite this long history of meteor spectroscopy, very few general surveys of meteor spectra have been presented. Harvey (1973) published statistics of visual inspection of 500 photographic meteor spectra. Borovička et al. (2005) presented a survey of 97 spectra of mainly sporadic meteors mostly in the magnitude range from +3 to 0 that were obtained by sensitive video technique. The spectra were classified according to the relative line intensities of $\mathrm{Mg}, \mathrm{Na}$, and $\mathrm{Fe}$, and three distinct populations of Na-free meteoroids were identified. We here extended that work by presenting a catalogue of 84 new video spectra of both sporadic and shower meteors. All these meteors were captured by our sensitive video technique.

Compared to photographic spectra of bright meteors, video spectra have a lower resolution and only contain a few lines. Only four meteoritic elements ( $\mathrm{Na}, \mathrm{Mg}, \mathrm{Ca}$, and $\mathrm{Fe}$ ) can be measured in most video spectra. Other observed emissions $(\mathrm{O}, \mathrm{N}$, and $\mathrm{N}_{2}$ ) are of atmospheric origin. On the other hand, the video technique is able to record spectra of relatively weak meteors (compared to photographic spectra). Thus we can obtain a more comprehensive sample of meteors for future analysis. Limits for our technique are +2 mag (the spectrum sufficiently bright for further analysis) and $-3.5 \mathrm{mag}$ (the signal is not yet saturated), corresponding to meteoroid sizes in the range of $1-10 \mathrm{~mm}$.

The meteor sample presented here contains members of all major meteor showers as well as sporadic meteors observed in 
Table 1. Station coordinates.

\begin{tabular}{l|ccc}
\hline \hline Station & Latitude & Longitude & Altitude $(m)$ \\
\hline $\begin{array}{l}\text { Spectral } \\
\text { Ondřejov }\end{array}$ & $\mathrm{N} 49^{\circ} 54^{\prime} 36.8^{\prime \prime}$ & E14 $46^{\prime} 48.75^{\prime \prime}$ & 524 \\
\hline $\begin{array}{l}\text { Second station } \\
\text { Kunžak }\end{array}$ & $\mathrm{N} 49^{\circ} 06^{\prime} 27.2^{\prime \prime}$ & $\mathrm{E} 15^{\circ} 12^{\prime} 2.755^{\prime \prime}$ & 652 \\
\hline $\begin{array}{l}\text { Spectral } \\
\text { Kurgan Tube }\end{array}$ & $\mathrm{N} 37^{\circ} 21^{\prime} 12.5^{\prime \prime}$ & $\mathrm{E} 68^{\circ} 46^{\prime} 18.3^{\prime \prime}$ & 425 \\
\hline $\begin{array}{l}\text { Second station } \\
\text { Gissar }\end{array}$ & $\mathrm{N} 38^{\circ} 29^{\prime} 23.3^{\prime \prime}$ & $\mathrm{E} 68^{\circ} 40^{\prime} 53.0^{\prime \prime}$ & 722 \\
\hline $\begin{array}{l}\text { Spectral } \\
\text { Brenna }\end{array}$ & $\mathrm{N} 45^{\circ} 44^{\prime} 01.3^{\prime \prime}$ & $\mathrm{E} 09^{\circ} 11^{\prime} 16.5^{\prime \prime}$ & 333 \\
\hline $\begin{array}{l}\text { Second station } \\
\text { Barengo }\end{array}$ & $\mathrm{N} 45^{\circ} 33^{\prime} 57.8^{\prime \prime}$ & $\mathrm{E} 08^{\circ} 30^{\prime} 18,2^{\prime \prime}$ & 238 \\
\hline
\end{tabular}

Notes. Most of the meteors we used here were observed from the base Ondřejov-Kunžak. The Leonids 09B17115, 09B17123, and 09B17192 were observed from the base Kurgan Tube-Gissar in Tajikistan. Only the meteor DRA06 was observed from the base Brenna-Barego in Italy during the Draconid campaign.

different parts of the year and different parts of the night. Our catalogue is therefore representative in the sense that it contains all common types of spectra in the given range of meteor brightness. In comparison with the work of Borovička et al. (2005), we used an image intensifier that provides a larger field of view, so that the spectral coverage was better. Our catalogue is intended to serve as a reference work for future spectral surveys of meteors.

\section{Observations and equipment}

Most observations were performed during the periods of activity of major meteor showers (Quadrantids, Lyrids, $\eta$ Aquarids, Perseids, Orionids, Leonids, and Geminids) in the years between 2006 and 2012. Most observations were carried out from the base Ondřejov-Kunžak. The distance between the stations is $92.5 \mathrm{~km}$. But several meteors were observed during observation campaigns in Tajikistan and Italy. For more details see Table 1.

Each station was equipped with S-VHS-C camcorders with the second-generation image intensifiers Mullard XX1332. One direct camera and one spectral camera was operated from the first station and one direct camera was operated from the second station. The spectral grating with 600 grooves $/ \mathrm{mm}$ and the Arsat $1.4 / 50 \mathrm{~mm}$ lens $\left(\mathrm{FOV} \approx 50^{\circ}\right)$ was used for all spectral observations, except for the 2011 Draconid campaign, when the lens Jupiter $2 / 85 \mathrm{~mm}\left(\mathrm{FOV} \approx 30^{\circ}\right)$ was used. The resulting dispersion was $30 \AA$ pixel $^{-1}$ for the Arsat lens and $15 \AA$ pixel $^{-1}$ for the Jupiter lens. The spectral sensitivity extends from $3800 \AA$ to $9000 \AA$ A. The sensitivity curve for the whole system (camera, image intensifier, and lens) is given in Fig. 1. The image intensifier affects the spectral sensitivity of the system by far the most, the individual differences between different lenses can be neglected. The intensity was normalized to unity at the wavelength $5500 \AA$. The Arsat $1.4 / 50 \mathrm{~mm}$ and the Jupiter 2/85 mm lenses were used for direct observations.

For our standard observations (from the base Ondřejov-Kunžak), video data were recorded by S-VHS video recorders on S-VHS video tapes (until the year 2008). After 2009, all videos were recorded straight onto the PC hard drive. Direct recording to the DV cassette was used during expeditions. These records were inspected using the automatic meteor-detection software MetRec (Molau 1999). Parts of the record, with detected meteors, were then saved as uncompressed

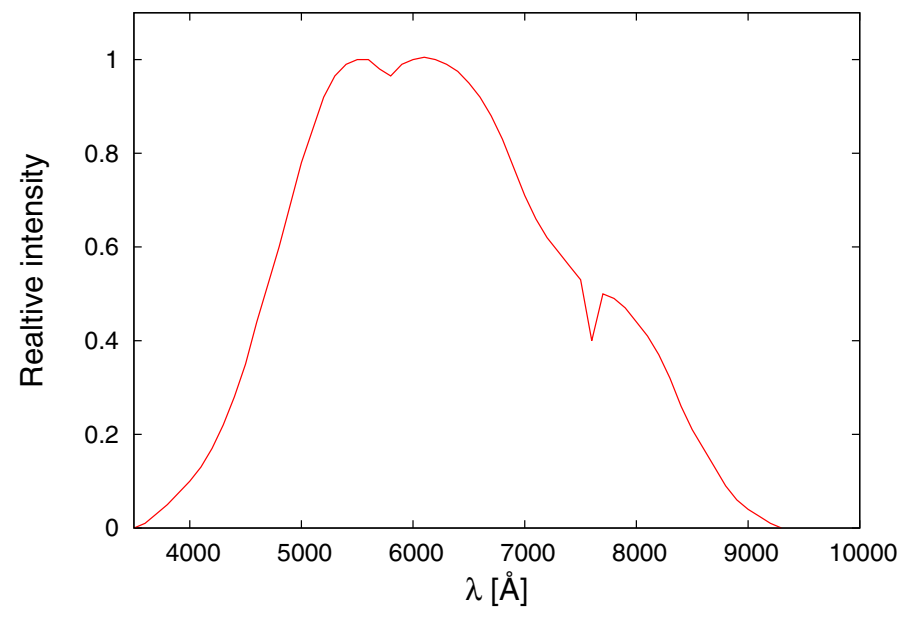

Fig. 1. Sensitivity of the spectral equipment (the S-VHS-C camera and the Mullard XX1332 image intensifier and lens) that we used for our observations. This calibration curve was obtained by measuring stellar spectra. The relative spectral intensity has been normalized to unity at $5500 \AA$. The dip at $7600 \AA$ is due to $\mathrm{O}_{2}$ atmospheric absorption.

AVI files with a resolution of $768 \times 576$ pixels $\times 8$ bit. The frame rate was 25 images per second, from which we obtained a time resolution of $0.04 \mathrm{~s}$. These 8 bit AVI files were then used for all measurements and data analysis presented in this paper.

We only selected meteors that were recorded from both stations for this paper and for which we managed to obtain good spectra. By "good" spectra we mean spectra where the entire meteor (or at least a significant part of it for very long meteors) appeared inside the field of view of the camera and at least the most important part of the spectrum (5000-8000 ̊) was covered. For the major showers we did not include all good spectra, but only representative ones (several meteors of different brightness).

\section{Data reduction}

The date of the observation of the meteor is encoded in the number of the corresponding meteor. The number is given in the form YYMDDXXX, where YY are the last two digits of the year. M is the month of the year: 1 to 9 - January to September and A - October, B - November and C - December. The digits DD 
V. Vojáček et al.: Catalogue of representative meteor spectra

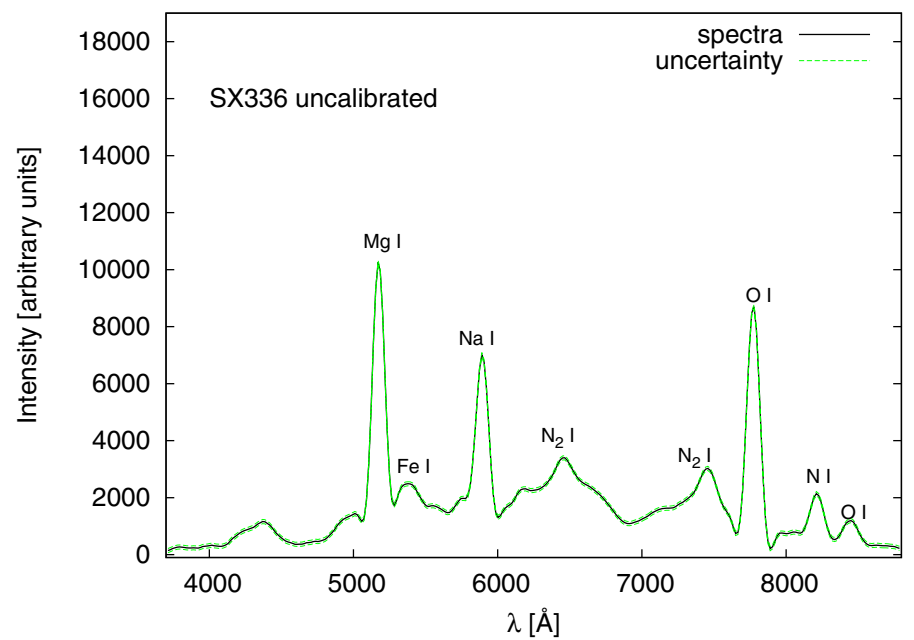

Fig. 2. Example of a spectrum (SX336) not calibrated for the spectral response of the instrument.

indicate the day when the observation started (the evening date). This means that the actual day of the observation is equal to DD if time $T>12 \mathrm{UT}$ and DD+1 if $T<12 \mathrm{UT}$. The last three digits $\mathrm{XXX}$ indicate the number of the meteor; the counting starts at the beginning of the night. For example, meteor 06C13137 was the 137 th metor observed on 13 December 2006. The only exception is the single Draconid meteor DRA06. This meteor was observed during the Draconid observation campaign in Northern Italy on 8 October of 2011. The geocentric radiant, the zenith distance of the apparent radiant, the beginning height, the terminal height, the maximum brightness, the photometric mass, the entry velocity, and the spectral type for each meteor are also included.

The name of the meteor spectra contains the prefix SX followed by the number of the spectrum. The numbering is carried out continuously in chronological order.

During the data reduction, we excluded the weakest meteors with a low signal-to-noise ratio. Finally, we used a total number of 84 meteors, 54 of which were sporadic meteors and 30 were showers meteors. Some of the sporadic meteors may be members of minor showers, but the association is unclear, and we counted them as sporadic in the following discussion.

The brightness and positions of all meteors were measured manually in each video frame with a special software developed by Borovička et al. (2005). By combining data from two direct cameras, we determined atmospheric trajectories and heliocentric orbits of meteoroids by the least-squares method (Borovička 1990). The Southworth-Hawkins D-criterion (Southworth \& Hawkins 1963) was used to determine the meteor shower membership. The threshold value for the D-criterion was arbitrarily chosen to be 0.2 , although none of the determined shower meteors had a value of the D-criterion higher than 0.15 . By knowing the meteoroid trajectories in the atmosphere, we were able to determine their absolute magnitude and photometric mass.

Video files with meteor spectra were processed with our standard procedure (Borovička et al. 2005). Positional and photometric calibration was made using the zero-order images of stars in the field. When each frame in which the spectrum appeared was scanned, we refined the wavelengths using wellknown atomic lines of the meteor emission.

In Figs. 2 and 3 we present one typical spectrum from the catalogue. In Fig. 2 the uncalibrated spectrum SX336 is given. The spectrum is calibrated for spectral sensitivity of the system

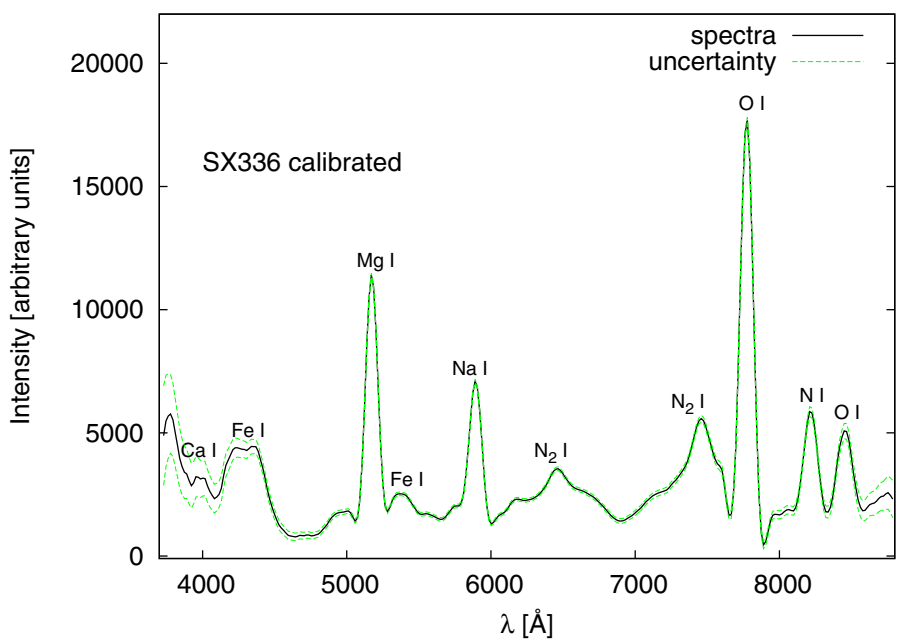

Fig. 3. Spectrum SX336 after correction for the spectral response of the instrument.

in Fig. 3. Below $4000 \AA$ and above $9000 \AA$, the final spectra can be strongly influenced by the noise after the calibration due to the low sensitivity of the equipment at these peripheral parts.

Statistical analysis of the noise in video frames was performed for selected recordings. We determined the average value of obtained standard deviations of the Gaussian noise for our equipment, $\sigma=14$ (in device arbitrary units). We then used this value to compute uncertainties for all spectra presented in the catalogue. Since spectra presented in the catalogue are the sums of individual frames, we determined the spectral uncertainties as the multiplication of the standard deviation and the square root of the number of frames used for a given spectrum. We applied the same relative uncertainties for the calibrated spectra.

For the basic analysis presented here, we also measured the spectral line intensities. This procedure is challenging since several components such as continuous emission, nitrogen bands, and other lines can contribute to the intensity of the peak at the line position. For this reason, we estimated intensities of all of these components that contribute to the resulting shape of the spectra to fit each spectrum completely. The fit parameters (e.g. spectral component intensities, temperature of the Planck emission, and the vibrational and rotational temperature of nitrogen bands) were changed manually by the operator of the software to fit the spectrum as well as possible. This procedure was done on each video frame, except for the temperature of Planck continuum and nitrogen bands, where one temperature for all frames was sufficient. In this way, we obtained spectral line intensities for each frame. The individual element intensities were then summed to find the total intensity for the corresponding multiplet.

We must point out that the spectral line intensities estimated by this procedure are meant to have rather illustrative purpose. We are not able, at this stage of the research, to determine uncertainties for line strengths or their ratios.

\section{Description and classification of the spectra}

\subsection{Description of the spectra}

The observed spectrum usually consists of the continuum, the emission from the heated atmosphere (specifically the oxygen and nitrogen lines and the nitrogen molecule bands) and the emission that originated in the evaporated material of the meteoroid. 
Table 2. Lines contributing to the meteor spectrum in the range of $3800 \AA$ Å-8700 ̊.

\begin{tabular}{|c|c|c|}
\hline $\begin{array}{c}\lambda \\
(\AA) \\
(\AA)\end{array}$ & Chemical species & Multiplet \\
\hline \multicolumn{3}{|c|}{ Low-temperature lines } \\
\hline 3831 & Mg I & 3 \\
\hline $3859,3885,3926$ & $\mathrm{Fe} \mathrm{I}$ & 4 \\
\hline $4045,4066,4143$ & $\mathrm{Fe} \mathrm{I}$ & 43 \\
\hline $4201,4273,4308,4326$ & $\mathrm{Fe} I$ & 42 \\
\hline 4226 & $\mathrm{Ca} \mathrm{I}$ & 2 \\
\hline 4255 & Cr I & 1 \\
\hline 4384,4405 & $\mathrm{Fe} \mathrm{I}$ & 41 \\
\hline 4703 & $\mathrm{Mg} \mathrm{I}$ & 11 \\
\hline $4872,4891,4920,4957,5008$ & $\mathrm{Fe} \mathrm{I}$ & 318 \\
\hline 5182 & Mg I & 2 \\
\hline $5269,5328,5371,5404,5431,5449$ & $\mathrm{Fe} \mathrm{I}$ & 15 \\
\hline 5892 & $\mathrm{Na} \mathrm{I}$ & 1 \\
\hline \multicolumn{3}{|c|}{ High-temperature lines } \\
\hline 3934,3969 & $\mathrm{Ca}$ II & 1 \\
\hline 4481 & Mg II & 4 \\
\hline 6347,6371 & Si II & 2 \\
\hline \multicolumn{3}{|c|}{ Wake lines } \\
\hline $4376,4427,4462,4482$ & $\mathrm{Fe} \mathrm{I}$ & 2 \\
\hline 4571 & Mg I & 1 \\
\hline 5169,5205 & $\mathrm{Fe} \mathrm{I}$ & 1 \\
\hline \multicolumn{3}{|c|}{ Atmospheric lines and bands } \\
\hline 5330 & OI & 12 \\
\hline $5700-6000$ & $\mathrm{~N}_{2}$ 1st. positive & $\Delta v=4$ \\
\hline $6200-6800$ & $\mathrm{~N}_{2}$ 1st. positive & $\Delta v=3$ \\
\hline $7000-7500$ & $\mathrm{~N}_{2}$ 1st. positive & $\Delta v=2$ \\
\hline 6157 & O I & 10 \\
\hline 6455 & O I & 9 \\
\hline 6484 & N I & 21 \\
\hline $7424,7442,7468$ & N I & 3 \\
\hline 7774 & O I & 1 \\
\hline $8186,8218,8143$ & N I & 2 \\
\hline 8446 & O I & 4 \\
\hline 8682 & $\mathrm{~N} \mathrm{I}$ & 1 \\
\hline \multicolumn{3}{|c|}{ Train lines } \\
\hline 5577 & {$[\mathrm{O} I]$} & $3 \mathrm{~F}$ \\
\hline
\end{tabular}

Notes. The lines are only given individually within the multiplet when they are well enough separated to be resolved in the video spectra. The multiplet numbers are given according to Moore (1945).

In Table 2 we show the list of lines that significantly contribute to the meteor spectra. According to their origin, these lines are divided into several groups. The lines of the first group are part of a low-temperature $(\approx 4500 \mathrm{~K})$ spectral component. The second group consists of lines of a high-temperature spectral component $(\approx 10000 \mathrm{~K})$. However, the lines of the second group are usually visible in bright and fast meteors and are of low importance for meteors within the magnitude range presented here. Another group consists of lines emitted just behind the meteoroid; they are prominent in meteor wakes. These lines only last a fraction of a second and are of the low-energy excitation intercombination origin and cannot be fitted by a thermal model (Borovička \& Jenniskens 2000). Although they are

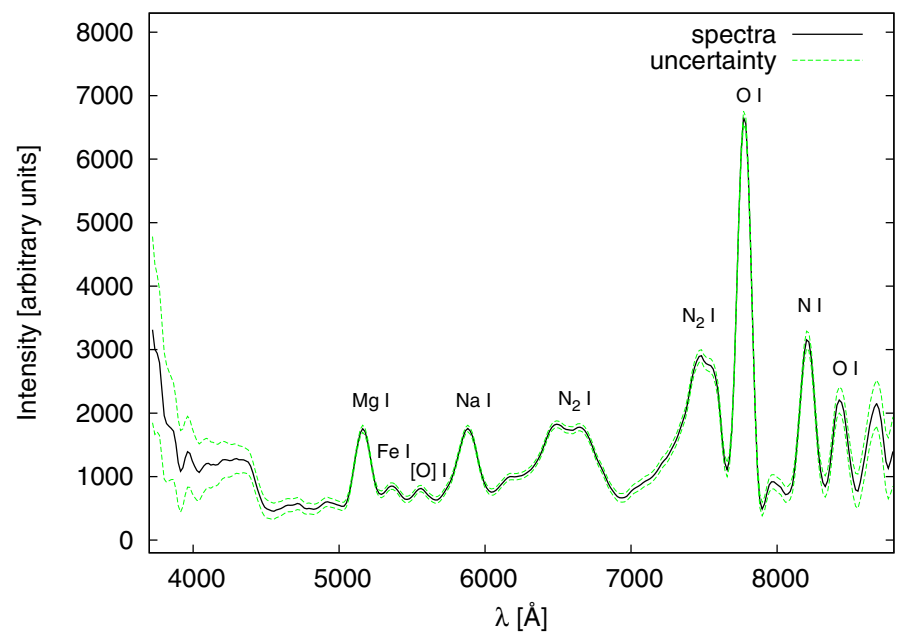

Fig. 4. Perseid spectrum SX1802. An example of a typical spectrum within the brightness range presented in the catalogue.

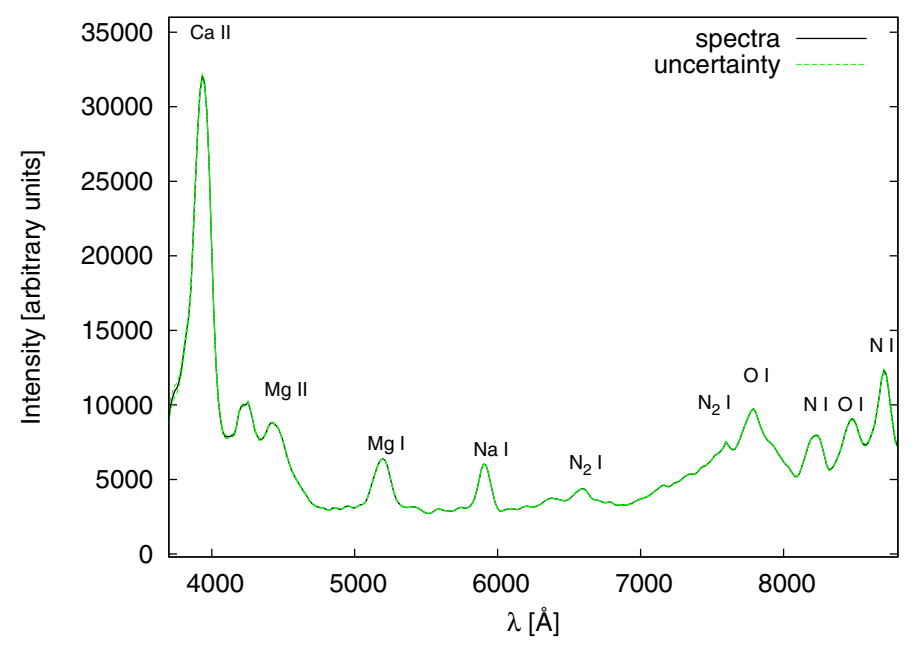

Fig. 5. Spectrum SX1837 of a bright Perseid. The meteor had a maximum brightness of -9.2 mag. Because the spectra were oversaturated on the video frames around the brightness maximum, one frame of the sequence was chosen. The brightness of the meteor in this frame was $-7.5 \mathrm{mag}$.

present typically in the meteor wake, they may also occur in the meteor heads, especially if the collisional deexcitation rate is low (Borovička et al. 2005). A significant part of meteor spectra is formed by lines and bands of atmospheric origin $(\mathrm{O}$ and $\mathrm{N}$ at $\approx 10000 \mathrm{~K})$. In the last group, there is only one line, the forbidden green oxygen line at $5577 \AA$. This line persists for nearly a second after the meteor disappears and forms a short-duration trail. This line is often noticeable in fast meteors.

The lines of the four meteoric elements can be recognized in the video spectra: magnesium (Mg I), sodium ( $\mathrm{Na}$ I), iron (Fe I), and calcium ( $\mathrm{Ca} \mathrm{I}$ ). However, the calcium lines are only sufficiently intense in bright meteors because our intensifier only has a low image sensitivity in the blue part of the spectrum (see Fig. 1). The Cr I lines are blended with Fe I and cannot be measured.

In meteors that are bright and fast enough, the lines of the high-temperature component (Ca II, Mg II, and Si II) may be present. In Figs. 4 and 5 spectra of two Perseid meteors $\left(\approx 60 \mathrm{~km} \mathrm{~s}^{-1}\right)$ of different brightness are compared. The maximum absolute magnitude of the meteor that formed the spectrum SX1802 in Fig. 4 was -1.6 mag. The only distinguishable meteoric lines are the low-temperature component lines $\mathrm{Mg} \mathrm{I}$, 


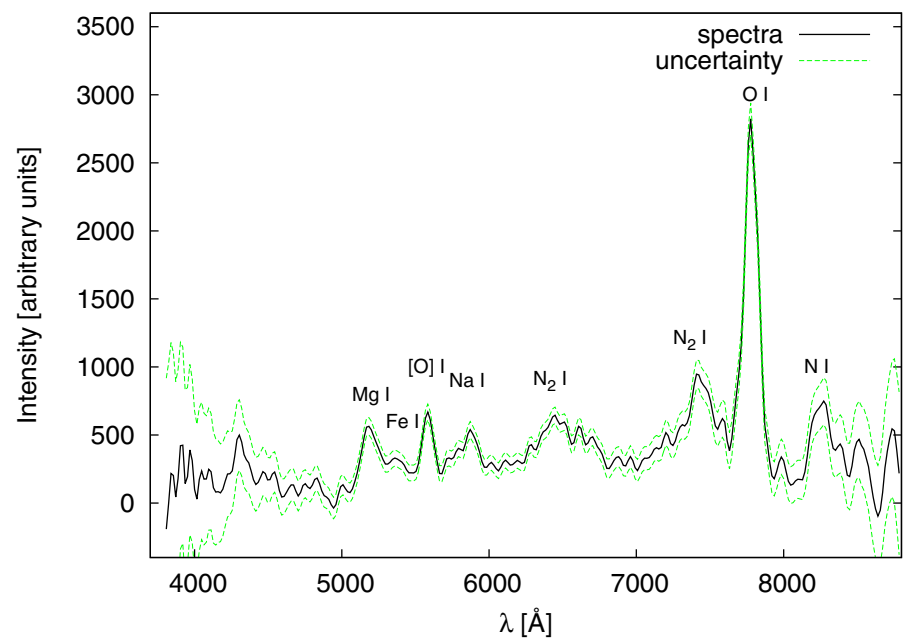

Fig. 6. Spectrum of SX457. An example of the spectrum of a fast meteor with a speed of $50.7 \mathrm{~km} \mathrm{~s}^{-1}$.

$\mathrm{Na}$ I, and Fe I. As an example of a bright Perseid meteor, the spectrum SX1837 is shown in Fig. 5. Because of the high maximum brightness of $-9.2 \mathrm{mag}$, the first order spectrum was oversaturated in video frames during the brightness maximum, therefore we did not include this spectrum in the catalogue (for more details about this exceptional meteor see Spurný et al. 2014). Moreover, the approach for the classification of spectra that we use below was only developed for small meteroids in the $1-10 \mathrm{~mm}$ size range. We chose only one frame from the sequence for this example where the spectra were not yet oversaturated. Low-temperature atomic lines are visible $(\mathrm{Mg} \mathrm{I}, \mathrm{Na}$ I, and $\mathrm{Fe} \mathrm{I}$ ), but the high-temperature atomic lines ( $\mathrm{Mg}$ II, Ca II) are also sufficiently bright in this case.

The appearance of the spectrum depends not only on the temperature, but also on the speed of the meteor. As an example of a spectrum of a fast $\left(50.7 \mathrm{~km} \mathrm{~s}^{-1}\right)$ meteor, the spectrum SX457 is given in Fig. 6. In contrast, in Fig. 7 the example of a slow $\left(24.5 \mathrm{~km} \mathrm{~s}^{-1}\right)$ meteor is presented (the spectrum SX1206). Both meteors had a similar visual magnitude $(\approx+1 \mathrm{mag})$.

The atmospheric lines of O I, N I, the nitrogen bands $\mathrm{N}_{2}$, and the forbidden green oxygen line [O I] are much brighter in fast meteors and cause brighter red and infrared parts of the spectrum of a fast meteor than in the spectrum of a slow meteor. The only atmospheric line, eminent in the spectrum SX1206, is the brightest oxygen line O I at $7774 \AA$.

\subsection{Classification of spectra}

Only three meteoric lines ( $\mathrm{Na} \mathrm{I,} \mathrm{Mg} \mathrm{I,} \mathrm{and} \mathrm{Fe} \mathrm{I)} \mathrm{can} \mathrm{be} \mathrm{ex-}$ amined in typical video spectra. Nevertheless, the differences in the intensities of these lines can reveal the different composition of individual meteoroids. We used the multiplet 15 at 5270-5450 A to measure Fe because our equipment is very sensitive in this spectral region (compared to the low sensitivity for Fe lines near $4400 \AA$ ), and we summed contributions of all lines of the multiplet. For $\mathrm{Na}$ and $\mathrm{Mg}$ the bright lines of multiplet 1 and multiplet 2, respectively, were used.

For this analysis we did not take the differential ablation into account. The contributions of individual multiplets were summed along the whole meteor path, and we only worked with total intensities.

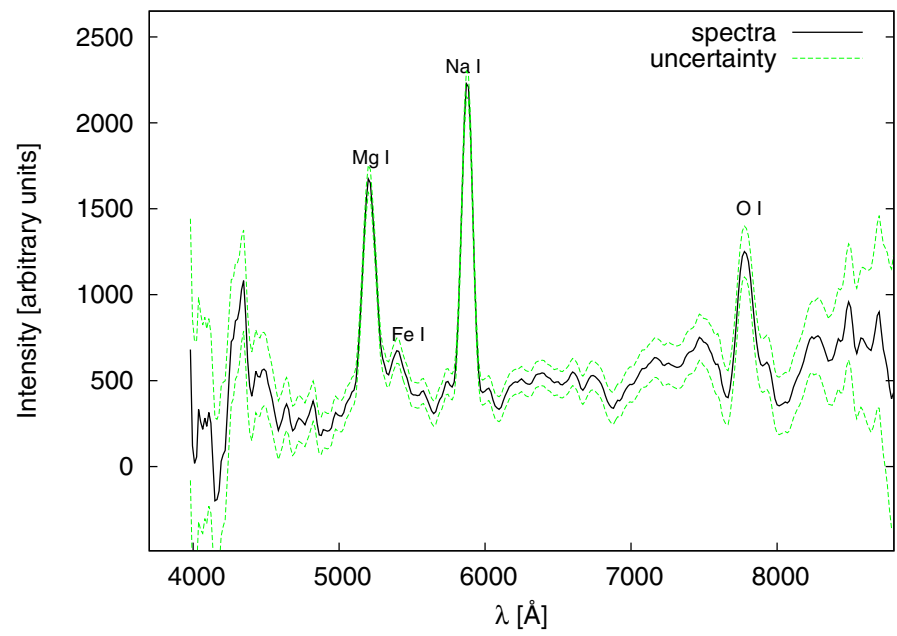

Fig. 7. Spectrum of SX1206. An example of a spectrum of a slow meteor with a speed of $24.5 \mathrm{~km} \mathrm{~s}^{-1}$.

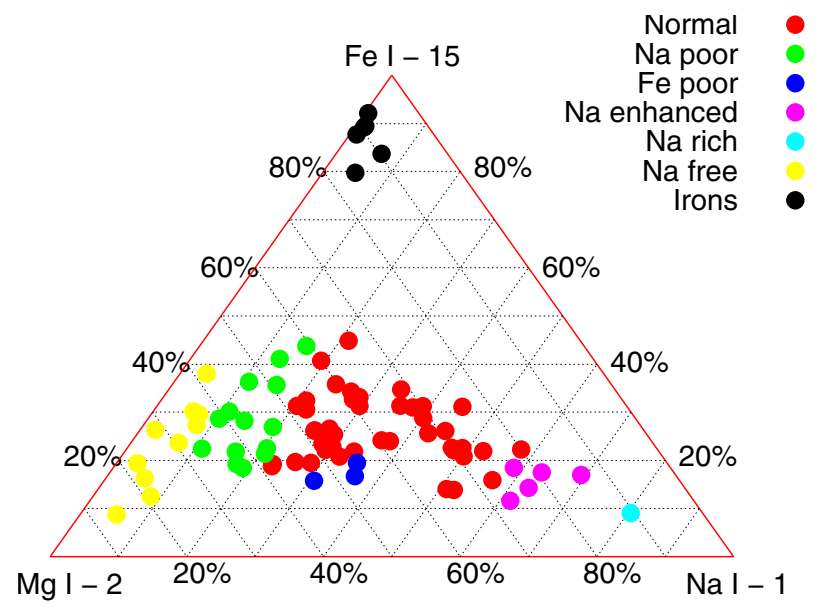

Fig. 8. Classification of meteor spectra. The ternary graph of the Mg I (2), Na I (1), and Fe I (15) multiplet relative intensities. Every group of meteoroids is represented with a different symbol.

To visualize the spectral classification we used the Mg-NaFe ternary diagram (Figs. 8 and 9) and the diagram of the dependence of the $\mathrm{Mg} / \mathrm{Na}$ ratio on speed (Fig. 10).

We used the spectral classification of meteor populations suggested by Borovička et al. (2005). The meteoroids were accordingly divided as follows.

\section{- Iron meteroids}

No sharp lines are present. Two bands formed by unresolved Fe multiplets (at 4200-4500 ̊ and 5100-5500 ̊). The Mg line at $5180 \AA$ can contribute to the intensity of the second band, but is much fainter than in normal spectra. The $\mathrm{Na}$ line is missing.

- Na-free meteoroids

No $\mathrm{Na}$ line but, not classified as iron meteorids. The $\mathrm{Fe} / \mathrm{Mg}$ ratio varies widely.

- Na-rich meteoroids

The spectrum is dominated by the $\mathrm{Na}$ line. The lines of $\mathrm{Mg}$ and $\mathrm{Fe}$ are present, but they are faint.

- Mainstream meteoroids

Mainstream meteoroids form the majority of meteoroids. Their spectra are closer to the expected chondritic spectra. But there are strong variations in the $\mathrm{Na}$ line intensity. Almost the whole range between the Na-free and Na-rich 


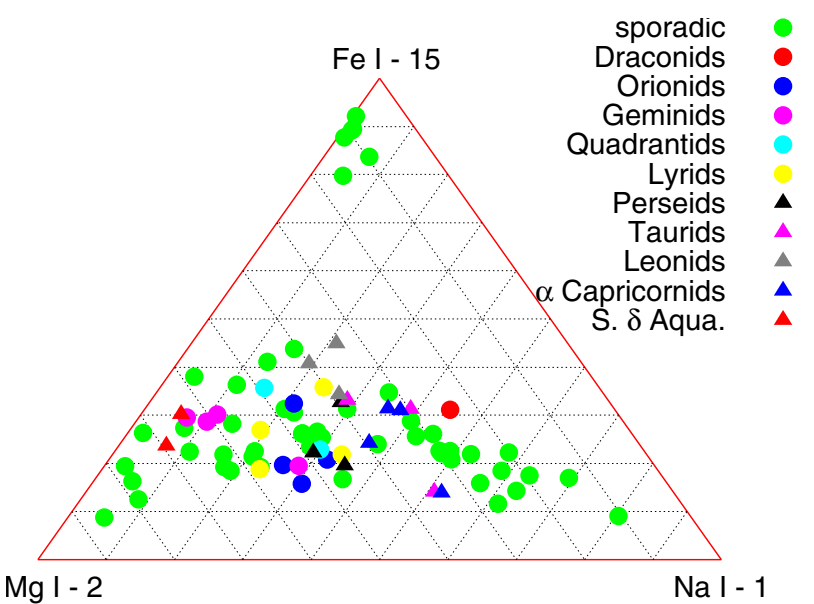

Fig. 9. Position of the meteor shower of meteoroids in the ternary graph of the Mg I (2), Na I (1), and Fe I (15) multiplet relative intensities. Every shower is represented with a different symbol.

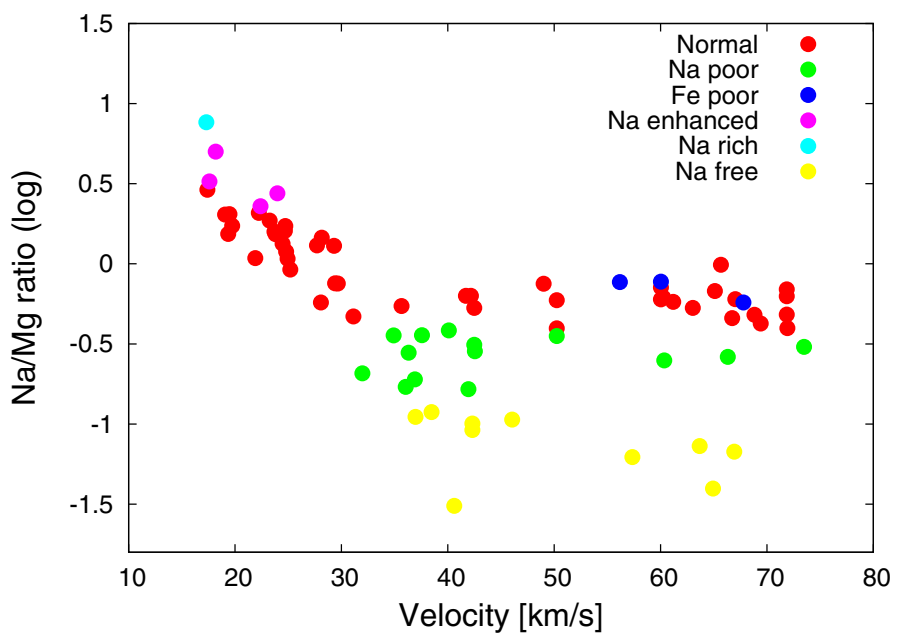

Fig. 10. Intensity ratio of the $\mathrm{Na} / \mathrm{Mg}$ lines in meteor spectra as a function of the meteor speed. Different spectral classes are plotted. Iron meteorids were excluded due to the high uncertainty in the determination of the line intensities of $\mathrm{Mg}$ and $\mathrm{Na}$ for this spectral class.

meteoroids is covered. The mainstream meteoroids are divided into four subclasses.

\section{- Normal meteoroids}

Normal meteoroids are defined as those lying near the expected position for chondritic bodies in the ternary diagram or with somewhat lower Fe intensity.

- Na-poor meteoroids

The Na line is weaker than expected for the given speed, but still reliably visible in contrast to Na-free meteoroids.

- Na-enhanced meteoroids

The Na line is significantly brighter than expected for the given speed, but not as dominant as for Na-rich meteoroids.

- Fe-poor meteoroids

Meteoroids with the expected $\mathrm{Na} / \mathrm{Mg}$ ratio, but with the Fe lines too faint to be classified as normal.

The spectrum SX1101 with dominant atmospheric lines and bands was excluded from the classification (see Fig. 12). The lines of $\mathrm{Na}, \mathrm{Mg}$, and $\mathrm{Fe}$ were not bright enough for the spectral analysis.

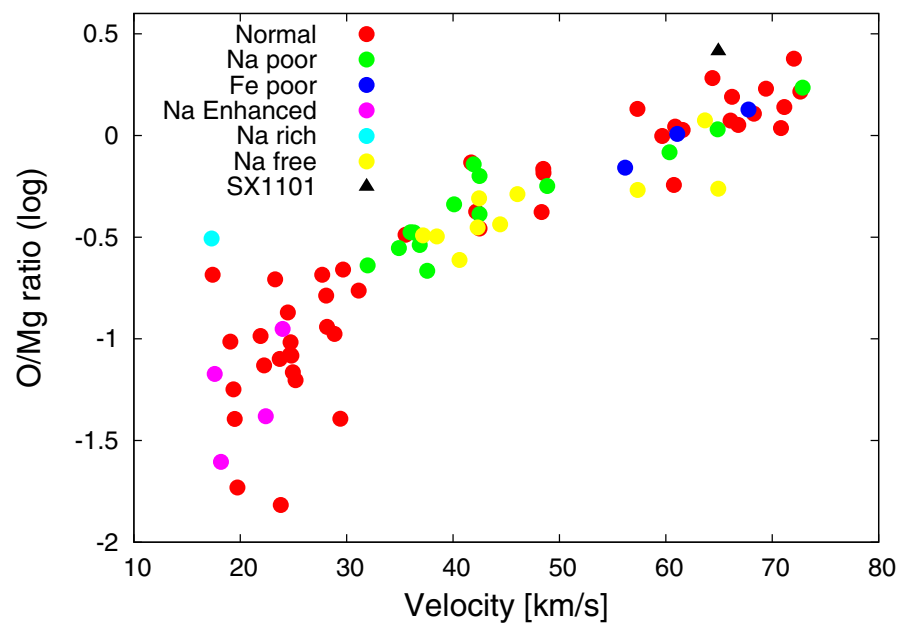

Fig. 11. Intensity ratio of the $\mathrm{O} / \mathrm{Mg}$ lines in meteors as a function of the meteor speed. Different spectral classes are plotted. The velocity uncertainties were negligible for display in the graph (for details see Table 4).

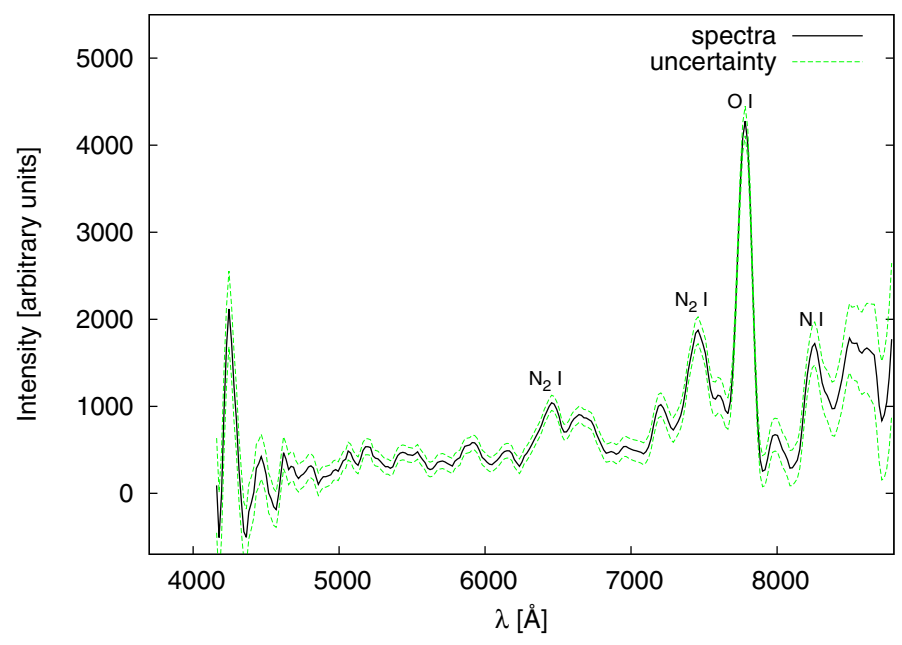

Fig. 12. Spectrum of SX1101 dominated by atmospheric emissions.

The decision to classify between Na-poor and Na-free meteoroids from the ternary graph was not always obvious (Fig. 8). For this purpose, Fig. 10 can be used, which shows the dependence of the $\mathrm{Na} / \mathrm{Mg}$ ratio on the velocity of the meteorid, where only intensities of $\mathrm{Mg}$ and $\mathrm{Na}$ are taken into account. A similar situation occurs between Fe-poor and normal meteoroids.

The intensities of the spectral lines correspond to different meteoroid compositions. But as we can see from spectral classification, the meteor speed also needs to be taken into account. According to Fig. 10, the $\mathrm{Na} / \mathrm{Mg}$ ratio increases for speeds below $35 \mathrm{~km} \mathrm{~s}^{-1}$. For speeds higher than $35 \mathrm{~km} \mathrm{~s}^{-1}$, the ratio is speed independent. Meteors classified as iron meteorids were excluded from Fig. 10, lines of $\mathrm{Na}$ and $\mathrm{Mg}$ are absent or weak for iron meteorids, and thus the determination of the $\mathrm{Na} / \mathrm{Mg}$ ratio is very inaccurate or even impossible.

In Fig. 11 we show the $\mathrm{O} / \mathrm{Mg}$ ratio as a function of velocity. The ratio of the $\mathrm{O}$ to $\mathrm{Mg}$ line intensities increases with the velocity. As mentioned before, fast meteors are characterized by more dominant atmospheric lines. For speeds below $30 \mathrm{~km} \mathrm{~s}^{-1}$ the scatter is large, mainly due to the faintness of the $\mathrm{O}$ line. Meteors classified as iron meteorids were excluded from Fig. 11.

This work is based on the sample of 84 meteors, which includes sporadic meteors and minor and major showers. When 


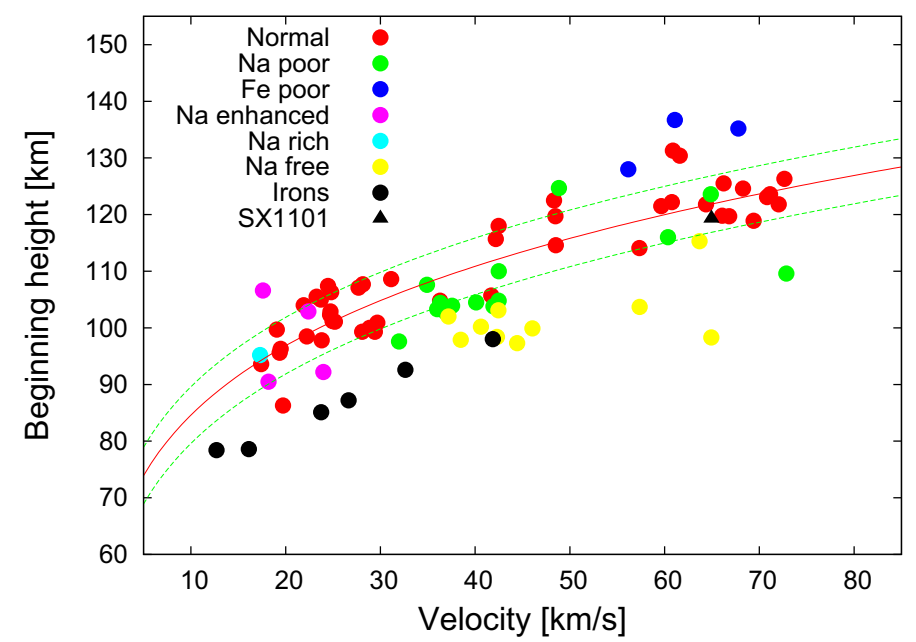

Fig. 13. Beginning height of meteor as a function of speed. Different spectral classes are plotted. The solid line shows the mean beginnings of meteoroids with average strengths. The dashed lines mark their limits $( \pm 5 \mathrm{~km})$. The equation of the empirical line is $H_{\mathrm{B}}=54 v^{0.195}$. Uncertainties for beginning heights and velocities were negligible for display in the graph (for details see Table 4).

compared to the work of Borovička et al. (2005), which was based on sample of 97 sporadic meteors and minor shower meteors, the results are similar.

Figure 9 shows Na-Mg-Fe ternary graph as well, but symbols of individual meteors represent the association with the shower. Most of the major shower meteoroids have been classified as normal. The only exceptions are meteoroids belonging to the Geminid shower and to the Southern $\delta$ Aquariids shower. Both Southern $\delta$ Aquariids meteoroids (SX731, SX738) were classified as Na-free. The Geminids have members of the Na-free, $\mathrm{Na}$-poor, and normal spectral groups. The reason of $\mathrm{Na}$ depletion in both showers is solar heating at low perihelion distances (Čapek \& Borovička 2009). The perihelion distance of Geminids is somewhat larger and the degree of $\mathrm{Na}$ depletion probably depends on the meteoroid size and structure, especially porosity (Borovička 2010).

It is well known that different strength categories of meteoroids in the millimeter-size range have different beginnings of the meteor luminous path (Ceplecha 1988). For a given speed, a meteoroid composed of stronger material has a lower beginning height than the meteoroid formed by weaker material. As we can see in Fig. 13, the iron meteoroids and the Na-free meteoroids started to ablate at lower heights than most meteoroids, while Fe-poor meteoroids started higher. Although Koten et al. (2003, 2004) found that the beginning height of cometary meteoroids also depends on the meteoroid mass, the dependence of the beginning height on the speed is sufficient for our purpose, and we can distinguish the variance of the material strength of different spectral classes of meteoroids.

\section{Meteoroid orbits}

The heliocentric orbits are known for all 84 meteors from double-station observations. The catalogue has a representative sample of orbits with a wide variety of orbital elements (see Fig. 14). Orbital elements such as the inclination $i$, the perihelion distance $q$, the argument of periapsis $\omega$, and the ascending node $\Omega$ are presented in the entire range of possible values. Higher values of eccentricity prevail mostly in the range from 0.6
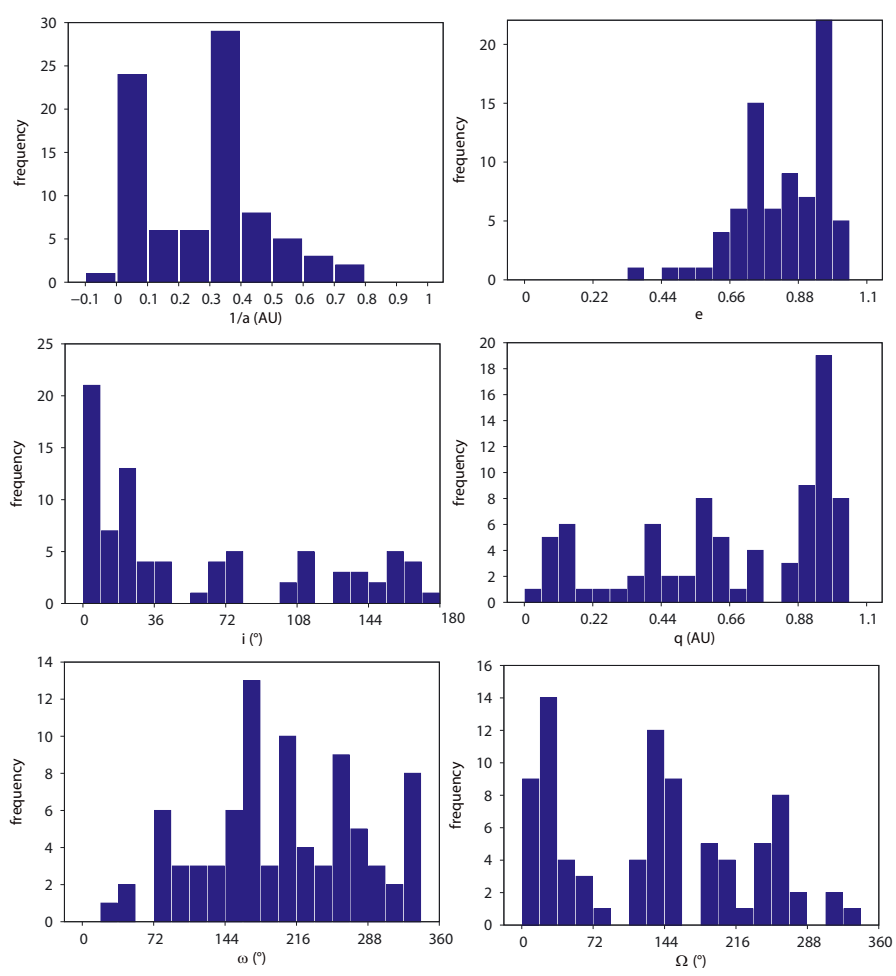

Fig. 14. Histograms of orbital elements of meteoroids in the catalogue of meteor spectra. $a$ is the major axis of the meteoroid, $e$ is the eccentricity, $i$ is the inclination, $q$ is the perihelion distance, $\omega$ is the argument of periapsis, and $\Omega$ is the ascending node.

to 1.0 , but this is obvious for bodies originating from comets and asteroids with usually higher values of eccentricity. There is also wide range of values of the semi-major axis $a$. Semi-major axes near the parabolic limit are hard to determine because they are sensitive to the determination of velocity, and so the accuracy is somewhat lower. One orbit is formally hyperbolic, but we believe this is due to a measurement error.

\subsection{Relation of meteor orbit to spectral classification}

We can use different sets of orbital parameters to classify meteor orbits: the perihelion distance $q$, the aphelion distance $Q$, the inclination $i$, and the Tisserand parameter relative to Jupiter $T_{\mathrm{J}}=$ $\left(a_{\mathrm{J}} / a\right)+2 \sqrt{\left(a / a_{\mathrm{J}}\right)\left(1-e^{2}\right)} \cos (i)$, where $a_{J}=5.2 \mathrm{AU}$ is the semimajor axis of Jupiter. These parameters are frequently used to determine typical orbits of asteroids, Jupiter family comets, and Halley-type comets.

Five classes of meteoroid orbits were defined by Borovička et al. (2005):

- (SA) Sun-approaching orbits: $q<0.2$ AU.

Orbits with small perihelion distances are defined as a separate class.

- (ES) Ecliptic shower orbits:

Members of ecliptical meteor showers. For example, the Taurid meteors derived from the comet 2P/Encke and other showers with orbits close to the boundary between asteroids and Jupiter family comets.

- (HT) Halley-type orbits: $T_{J}<2$ or $2<T_{J}<3$ and $i>45^{\circ}$.

- (JF) Jupiter-family orbits: $2<T_{J}<3$ and $i<45^{\circ}$ and $Q>4.5$ AU.

- (A-C) Asteroidal-chondritic orbits: $T_{J}>3$ or $Q<4.5 \mathrm{AU}$. 
Table 3. Orbital elements of meteoroids classified as iron meteorids.

\begin{tabular}{cccccccccc}
\hline \hline Spectra & $\begin{array}{c}a \\
(\mathrm{AU})\end{array}$ & $\begin{array}{c}q \\
(\mathrm{AU})\end{array}$ & $\begin{array}{c}Q \\
(\mathrm{AU})\end{array}$ & $\begin{array}{c}i \\
\left({ }^{\circ}\right)\end{array}$ & $\begin{array}{c}\omega \\
\left({ }^{\circ}\right)\end{array}$ & $\begin{array}{c}\Omega \\
\left({ }^{\circ}\right)\end{array}$ & $\begin{array}{c}v_{g} \\
\left(\mathrm{~km} \mathrm{~s}^{-1}\right)\end{array}$ & $T_{J}$ & shower \\
\hline SX001 & 2.5 & 0.110 & 5.0 & 28.9 & 325.1 & 16.95 & 40.3 & 2.4 & $\mathrm{SPO}$ \\
& 0.1 & 0.004 & 0.2 & 0.9 & 0.7 & - & 0.2 & 0.3 & \\
SX393 & 1.59 & 0.976 & 2.19 & 6.4 & 194.1 & 262.71 & 6.5 & 4.3 & $\mathrm{SPO}$ \\
& 0.02 & 0.001 & 0.05 & 0.2 & 0.3 & - & 0.2 & 0.2 & \\
SX689 & 1.84 & 0.966 & 2.72 & 41.3 & 150 & 45.77 & 24.1 & 3.6 & SPO \\
& 0.04 & 0.002 & 0.02 & 0.3 & 1 & - & 0.2 & 0.2 & \\
SX692 & 2.1 & 0.999 & 3.2 & 16 & 194 & 45.80 & 11.8 & 3.5 & SPO \\
& 0.2 & 0.004 & 0.4 & 1 & 3 & - & 0.8 & 0.6 & \\
SX1114 & 1.74 & 0.554 & 2.92 & 6.7 & 94.9 & 55.43 & 20.9 & 3.8 & SPO \\
& 0.05 & 0.006 & 0.09 & 0.2 & 0.09 & - & 0.2 & 0.08 & \\
SX1194 & 2.53 & 0.335 & 4.7 & 3.1 & 295.6 & 19.91 & 30.6 & 2.7 & SPO \\
& 0.07 & 0.003 & 0.1 & 0.2 & 0.4 & - & 0.2 & 0.05 & \\
\hline
\end{tabular}

Notes. The second row for each meteor contains the corresponding errors.

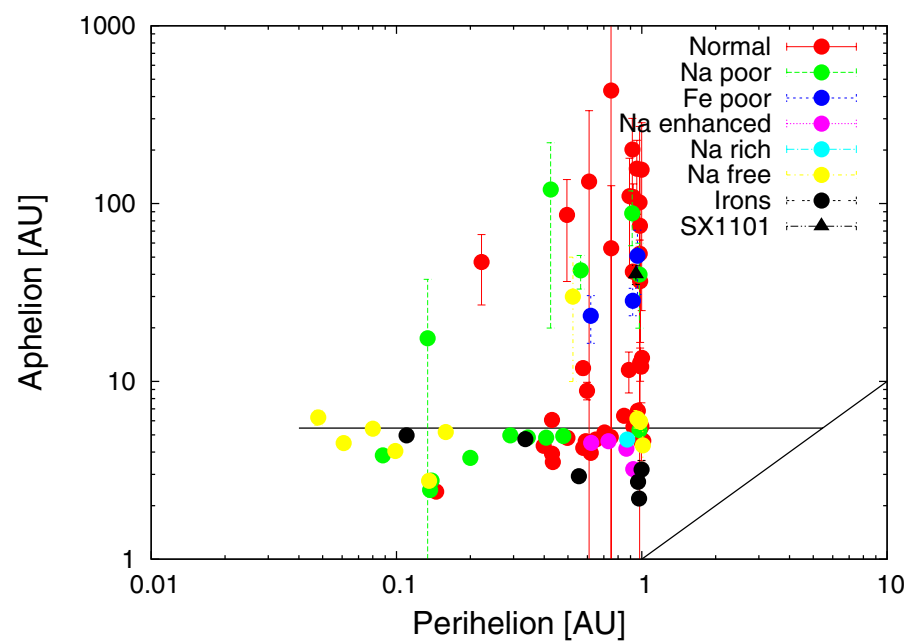

Fig. 15. Perihelion versus aphelion graph. Plot with error bars. Different symbols represent individual spectral classes. The solid line represents Jupiter's aphelion $Q_{J}=5.5 \mathrm{AU}$.

Spectral classes of meteoroids from the catalogue and their positions within the orbit classification schemes are shown in Figs. 15 and 16. Figure 15 shows the perihelion and the aphelion positions of meteoroids. Figure 16 shows the Tisserand parameter versus inclination.

We can now combine the spectral, orbital, and material strength classification and discuss the individual spectral classes separately.

\subsection{Iron meteorids}

The orbital elements of all six meteors from the catalogue that were classified as iron meteorids are shown in Table 3. Four of them (SX393, SX689, SX692, and SX1114) satisfy the condition for the asteroidal-chondritic class, their aphelion distances $Q$ are below 4.5 AU and their Tisserand parameters $T_{J}$ are greater than 3 . The meteoroid SX001 with a perihelion of only 0.11 AU can be classified as a Sun-approaching meteoroid. The orbit of meteoroid SX1194 can be classified as a Jupiter-family orbit, but asteroidal origin cannot be excluded, since the inclination is only 3 degrees and the aphelion of $4.7 \mathrm{AU}$ is not particularly high.

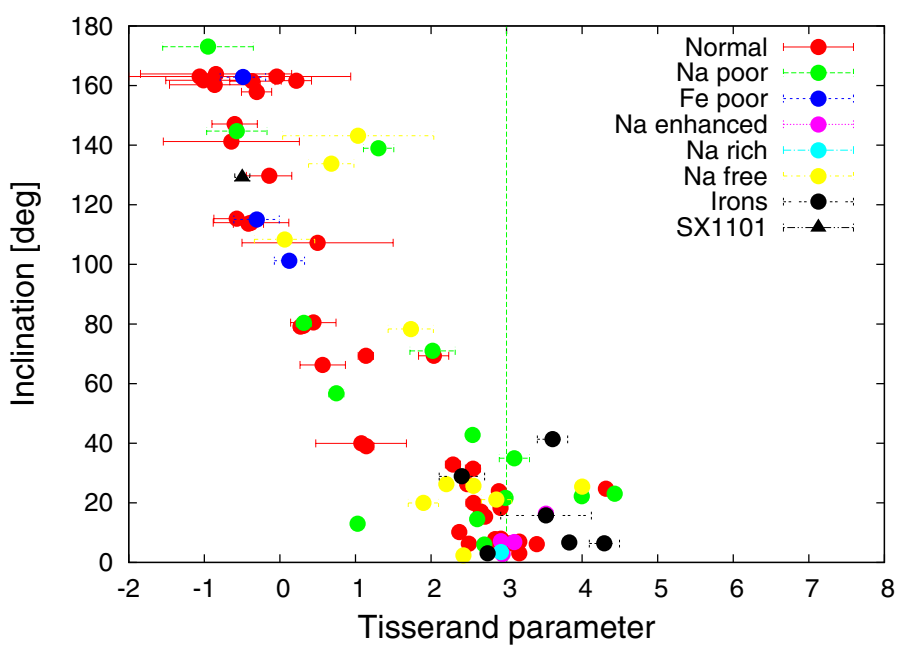

Fig. 16. Tisserand parameter versus inclination plot with error bars. Different symbols represent individual spectral classes. The dashed vertical line marks the Tisserand parameter $T_{J}=3$.

\subsection{Na-free meteoroids}

We can clearly distinguish two different populations in the Nafree meteoroids: the Sun-approaching population with small perihelions and the Halley-type population with high inclinations.

\subsubsection{Sun-approaching meteoroids}

Most of meteoroids with a perihelion distance $q<0.2$ are Na-free or Na-poor. The only exception is the iron meteroidtype meteoroid SX001 mentioned earlier, which, nevertheless, does not contain $\mathrm{Na}$ either. This corresponds to the conlcusion of Borovička et al. (2005), that frequent approaches to within $\approx 0.2$ AU to the Sun lead to the loss of $\mathrm{Na}$ from meteoroids in the millimeter-size range, irrespective to their origin (see also Čapek \& Borovička 2009).

The material of Na-free meteoroids also tends to have a greater strength (see Fig. 13).

The members of $\delta$ Aquariids $(q=0.07)$ can be found among the Na-free meteoroids. One member of the Geminid shower (the SX337) was also classified as Na-free. However, members of this stream (with a small perihelion distance $q=0.14 \mathrm{AU}$ ) have a wide spread of Na-line intensities. We can find Na-poor and also normal types of spectra within this meteor stream. The 
explanation is, as suggested by Borovička et al. (2005), that the $\mathrm{Na}$ content is correlated with the age of the meteoroid. Younger meteoroids that have suffered fewer passages close to the Sun retain more $\mathrm{Na}$, which implies that the Geminid meteoroid stream was not formed in one instant. A later analysis of Borovička et al. (2010) suggested that differences in porosity may be the main reason of the different $\mathrm{Na}$ content in the Geminids.

\subsubsection{Cometary Na-free meteoroids}

The close approach to the Sun is not the only process that causes depletion of $\mathrm{Na}$ in meteoroids. In our sample we have three meteoroids without a $\mathrm{Na}$ line in their spectra (SX350, SX696, and SX1104), but their orbits are different from those of Sunapproaching meteoroids. Their perihelion distances are closer to $1 \mathrm{AU}$, and they have high inclinations or even retrograde orbits. These orbits are of Halley type. According to Borovička et al. (2005), the reason for $\mathrm{Na}$ depletion in these types of orbits might be the long exposure to cosmic rays on the comet surface during their residence in the Oort cloud. This process can lead to the formation of Na-free refractory crust. The gradual or sudden disintegration of the crust during the cometary passage through the inner solar system then produces millimeter-sized compact Na-free meteoroids.

\subsection{Na-rich meteoroids}

There is only one Na-rich meteoroid (SX150) in our catalogue. The body has a Jupiter-family orbit.

\subsection{Normal meteoroids}

Both cometary and asteroidal orbits are found among meteorids classified as normal. But only part of these meteoroids have a typically chondritic composition, many of them show somewhat fainter Fe lines. According to the computation of the chondritic composition and the Halley dust composition, based on the data of Leonid observations, Borovička et al. (2005) assumed that the sample of meteoroids classified as normal is a mixture of normal chondritic material and cometary material similar to Leonids. Three Taurid meteors (SX263, SX1122, and SX1128) with ecliptic shower orbits have a normal composition.

\subsection{Fe-poor meteoroids}

Three of the meteoroids in the catalogue (SX211, SX1064, and SX1802) were classified as Fe-poor. The iron lines were too faint to classify the meteoroids as normal, although the boundary is somewhat arbitrary. All of the Fe-poor meteoroids have cometary Halley-type orbits.

Fe-poor meteorids have low material strength, their beginnings of ablation are usually high (see Fig. 13).

\subsection{Na-poor meteoroids}

Na-poor meteoroids are the transition between normal and $\mathrm{Na}$ free meteoroids. Like the Na-free meteorids, some of them have low perihelia, others have cometary orbits. Thus they probably have the same two origins as the Na-free meteoroids.

\subsection{Enhanced-Na meteoroids}

Five meteoroids (SX143, SX500, SX785, SX820, and SX983) were classified as enhanced-Na meteoroids. SX500 has a typical asteroidal-chondritic orbit. The other four meteoroids can be classified as ecliptical or Jupiter-family meteoroids. SX143, SX820, and SX983 were sporadic meteors. Their orbits were similar to the Na-rich meteoroid SX150. Borovička et al. (2008) studied enhanced-Na sporadic meteoroid SX498, and according to their analysis, this meteoroid had a Jupiter-family orbit.

\section{Catalogue}

The atmospheric trajectories and orbital elements for all meteors of the catalogue are shown in Tables 4 and 5.

The second row in Table 4 for each meteor contains the corresponding errors of the least-squares regression. The standard error for meteor heights are of about $10^{-2} \mathrm{~km}$. For the absolute magnitude $M_{\max }$, the standard error for our technique is $\approx 0.5 \mathrm{mag}$. The photometric mass is highly affected by the selected luminous efficiency. We used the luminous efficiency of Pecina \& Ceplecha (1983).

Heliocentric orbits are known for all meteors. In Table 5 we present orbital elements (reciprocal semi-major axis $1 / a$, eccentricity $e$, inclination $i$, perihelion distance $q$, aphelion distance $Q$, argument of periapsis $\omega$, longitude of the ascending node $\Omega$, Tisserand parameter $T_{\mathrm{J}}$, and geocentric velocity $v_{\mathrm{g}}$ ) for each meteor of the catalogue. Angular elements are given for the equinox J2000.0. The shower membership is given in the last column, SPO stands for sporadic meteor.

The spectra are plotted in Figs. 17-30 (available in electronic version only). The measurements of all frames of the spectra were combined, and the intensities summed along the whole meteor paths are given as a function of wavelength. Calibrated spectra are plotted. Calibrated and uncalibrated spectra of all 84 meteors are available at the CDS. The total intensities of the multiplets $\mathrm{Mg} 1-2, \mathrm{Na1}-1$, and Fe1-15 are given explicitly in a separate file.

\section{Conclusions}

We presented a survey of 84 meteors in the magnitude range from +2 to -3 , corresponding to meteor sizes from $1 \mathrm{~mm}$ to $10 \mathrm{~mm}$. Using parallel double-station observations, we also computed heliocentric orbits for all of these 84 meteors. We classified the meteor spectra according to the classification suggested by Borovička et al. (2005) based on the intensities of the low-temperature emission lines of $\mathrm{Na}, \mathrm{Mg}$, and Fe. Only part of meteoroids were found to have a typical chondritic composition. We found a variety of $\mathrm{Na}$ depletion, Fe depletion, or Na enhancement. Approximately $20 \%$ of the whole sample of millimeter-sized meteors was found to contain no sodium in the spectra. Three populations can be distinguished among the Nafree meteoroids: the iron meteoroids with an asteroidal origin, the Sun-approaching meteoroids with $\mathrm{Na}$ depleted by frequent approaches within $\approx 0.2$ AU to the Sun, and the cometary Nafree meteoroids with $\mathrm{Na}$ depletion that might be caused by long exposure to cosmic rays of the surface of comets in the Oort cloud.

Most of the major shower meteors have been classified as normal. Some members of the Geminid shower and the members of Southern $\delta$ Aquariids were classified as Na-free. The solar heating at low perihelia distances is the reason for the Na depletion of these showers (Čapek \& Borovička 2009). The variations 
of Na content among the Geminid meteor stream was confirmed. The differences in porosity may be the main reason of this effect (Borovička 2010).

Most of the meteoroids on the asteroidal-chondritic orbits were found to be iron meteoroids. This is in contrast to centimeter-sized and larger meteoroids where chondritic composition prevails, but it agrees with results of Borovička et al. (2005), who reported that millimeter-sized stony debris is rare. One iron meteoroid has a typical sun-approaching orbit.

Meteoroids with cometary origin had a heterogeonous composition, from $\mathrm{Na}$-free, $\mathrm{Na}$-poor, and $\mathrm{Fe}$-poor for Halley-type orbits to Na-rich and enhanced-Na for Jupiter-family orbits.

Acknowledgements. This work was supported by grant No. P209/11/1382 from GA ČR and by the project SVV-260089 by the Charles University in Prague.

\section{References}

Borovička, J. 1990, Bull. Astron. Inst. Czechoslovakia, 41, 391 Borovička, J. 1994a, A\&AS, 103, 83

Borovička, J. 1994b, Planet. Space Sci., 42, 145

Borovička, J. 2010, in Proc. International Meteor Conference, 26th IMC, Bareges, France, 2007, eds. J. Rendtel, \& J. Vaubaillon, 42

Borovička, J., \& Boček, J. 1995, Earth Moon and Planets, 71, 237

Borovička, J., \& Jenniskens, P. 2000, Earth Moon and Planets, 82, 399

Borovička, J., Koten, P., Spurný, P., Boček, J., \& Štork, R. 2005, Icarus, 174, 15

Borovička, J., Koten, P., Spurný, P., \& Štork, R. 2008, Earth Moon and Planets, 102,485
Borovička, J., Koten, P., Spurný, P., et al. 2010, in IAU Symp. 263, eds., J. A. Fernandez, D. Lazzaro, D. Prialnik, \& R. Schulz, 218

Ceplecha, Z. 1971, Bull. Astron.1 Inst. Czechoslovakia, 22, 219

Ceplecha, Z. 1988, Bull. Astron.1 Inst. Czechoslovakia, 39, 221

Ceplecha, Z., Borovička, J., Elford, W. G., et al. 1998, Space Sci. Rev., 84, 327 Čapek, D., \& Borovička, J. 2009, Icarus, 202, 361

Halliday, I. 1961, A Study of Spectral Line Identifications in Perseid Meteor Spectra, Publications of the Dominion Observatory, Ottawa (Duhamel)

Harvey, G. A. 1973, NASA Spec. Pub., 319, 131

Hemenway, C. L., Swider, A., \& Bowman, C. 1971, Can. J. Phys., 49, 1361

Koten, P., Spurný, P., Borovička, J., et al. 2003, in Proc. 2002 international science symposium on the Leonid meteor storms

Koten, P., Borovička, J., Spurný, P., Betlem, H., \& Evans, S. 2004, A\&A, 428, 683

Millman, P. M. 1963, Smithsonian Contributions to Astrophysics, 7, 119

Millman, P. M. 1980, in Solid Particles in the Solar System, eds. I. Halliday, \& B. A. McIntosh, IAU Symp., 90, 121

Millman, P. M., \& Clifton, K. S. 1975, Can. J. Phys., 53, 1939

Molau, S. 1999, in Proc. International Meteor Conference, 17th IMC, Stara Lesna, Slovakia, 1998

Moore, C. E. 1945, Contributions from the Princeton University Observatory, 20,1

Mukhamednazarov, S., \& Maltseva, N. V. 1989, Astronomicheskii Vestnik, 23, 297

Pecina, P., \& Ceplecha, Z. 1983, Bull. Astron.1 Inst. Czechoslovakia, 34, 102

Southworth, R. B., \& Hawkins, G. S. 1963, Smithsonian Contributions to Astrophysics, 7, 261

Spurný, P., Shrbený, L., Borovička, J., et al. 2014, A\&A, 563, A64

Tors, S., \& Orchiston, W. 2009, J. Astronomical History and Heritage, 12, 211

Zender, J., Koschny, D., Witasse, O., et al. 2004, in Proc. the International Meteor Conference, 22nd IMC, Bollmannsruh, Germany, 2003, eds. M. Triglav-Čekada, \& C. Trayner, 163 
V. Vojáček et al.: Catalogue of representative meteor spectra

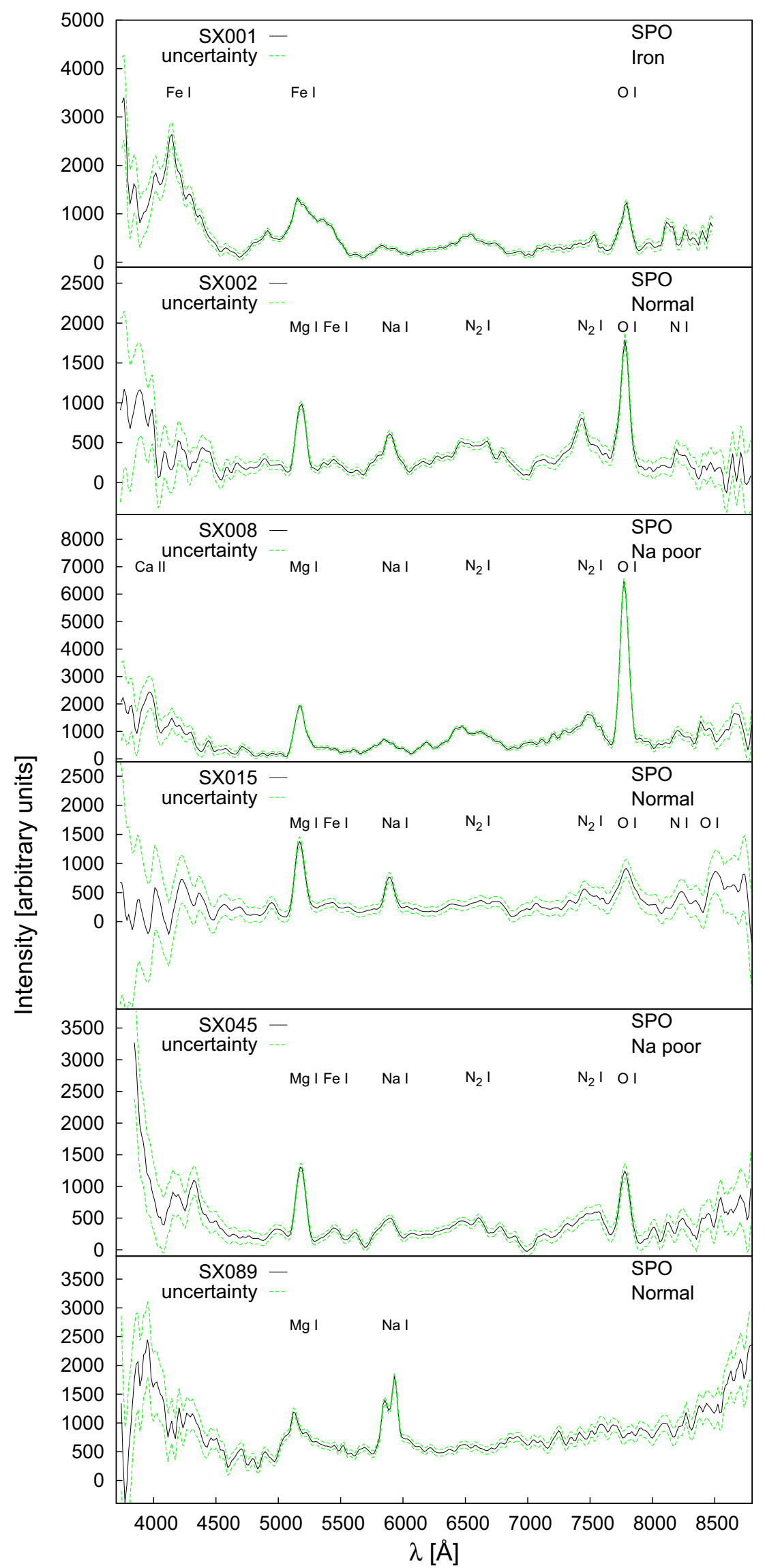

Fig. 17. Individual spectra of the catalogue, their spectral classification and shower identifications (SPO - sporadic meteors). Main lines and emission bands are identified. 
A\&A 580, A67 (2015)

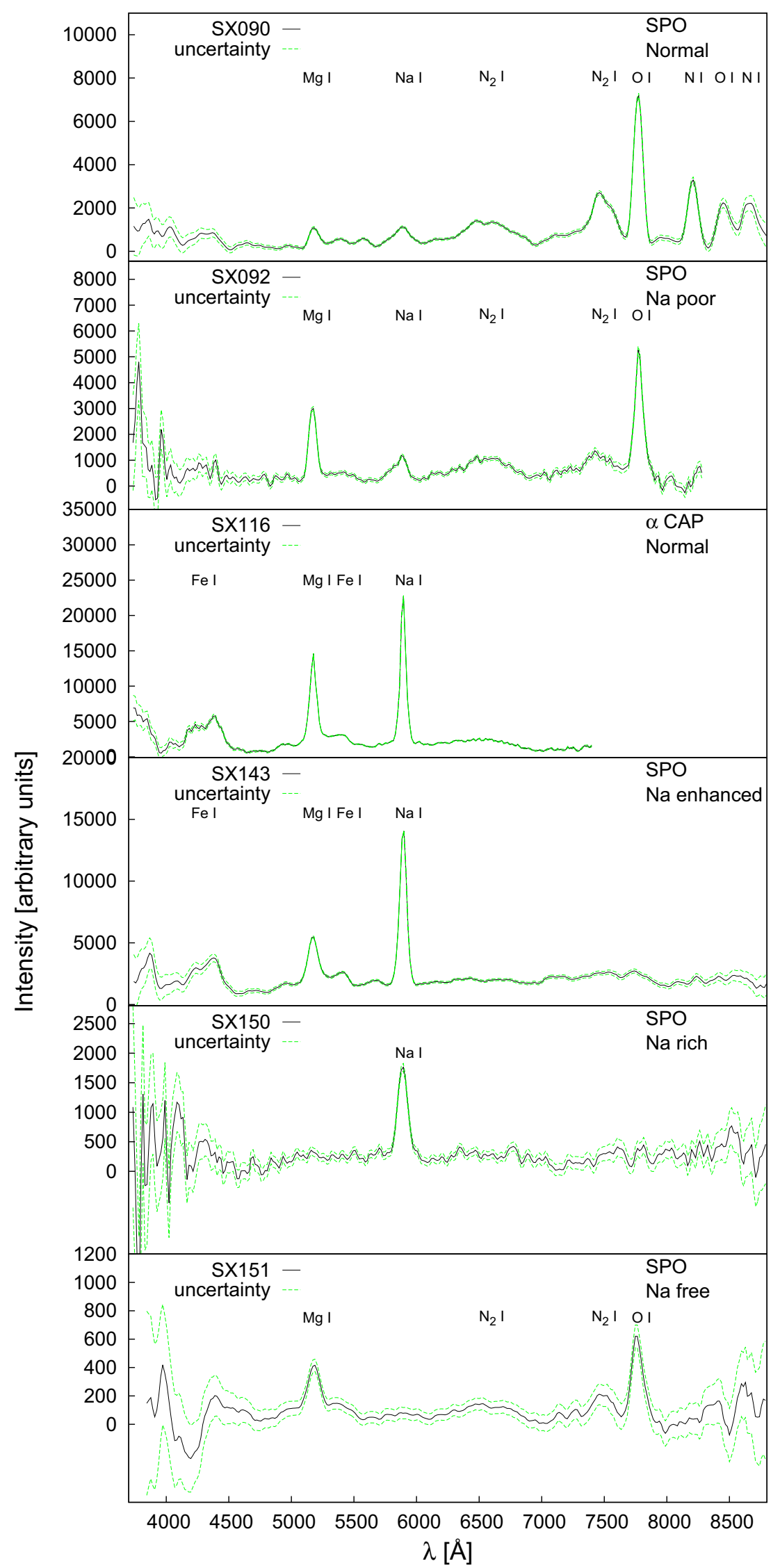

Fig. 18. Individual spectra of the catalogue, their spectral classification and shower identifications (SPO - sporadic meteors, $\alpha$ CAP $\alpha$ Capricornids). Main lines and emission bands are identified. 
V. Vojáček et al.: Catalogue of representative meteor spectra

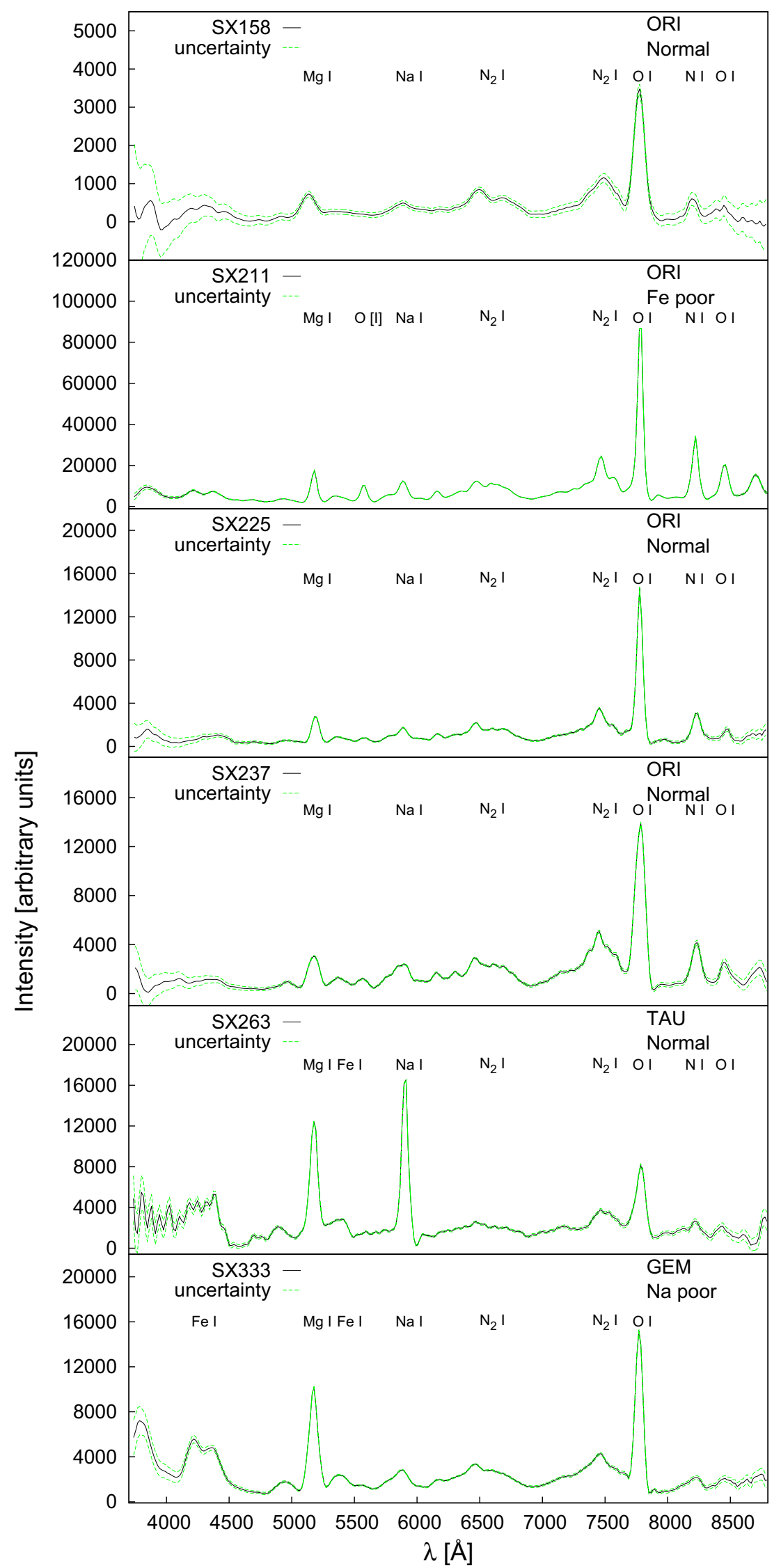

Fig. 19. Individual spectra of the catalogue, their spectral classification and shower identifications (ORI - Orionids, TAU - Taurids, GEM Geminids). Main lines and emission bands are identified. 
A\&A 580, A67 (2015)

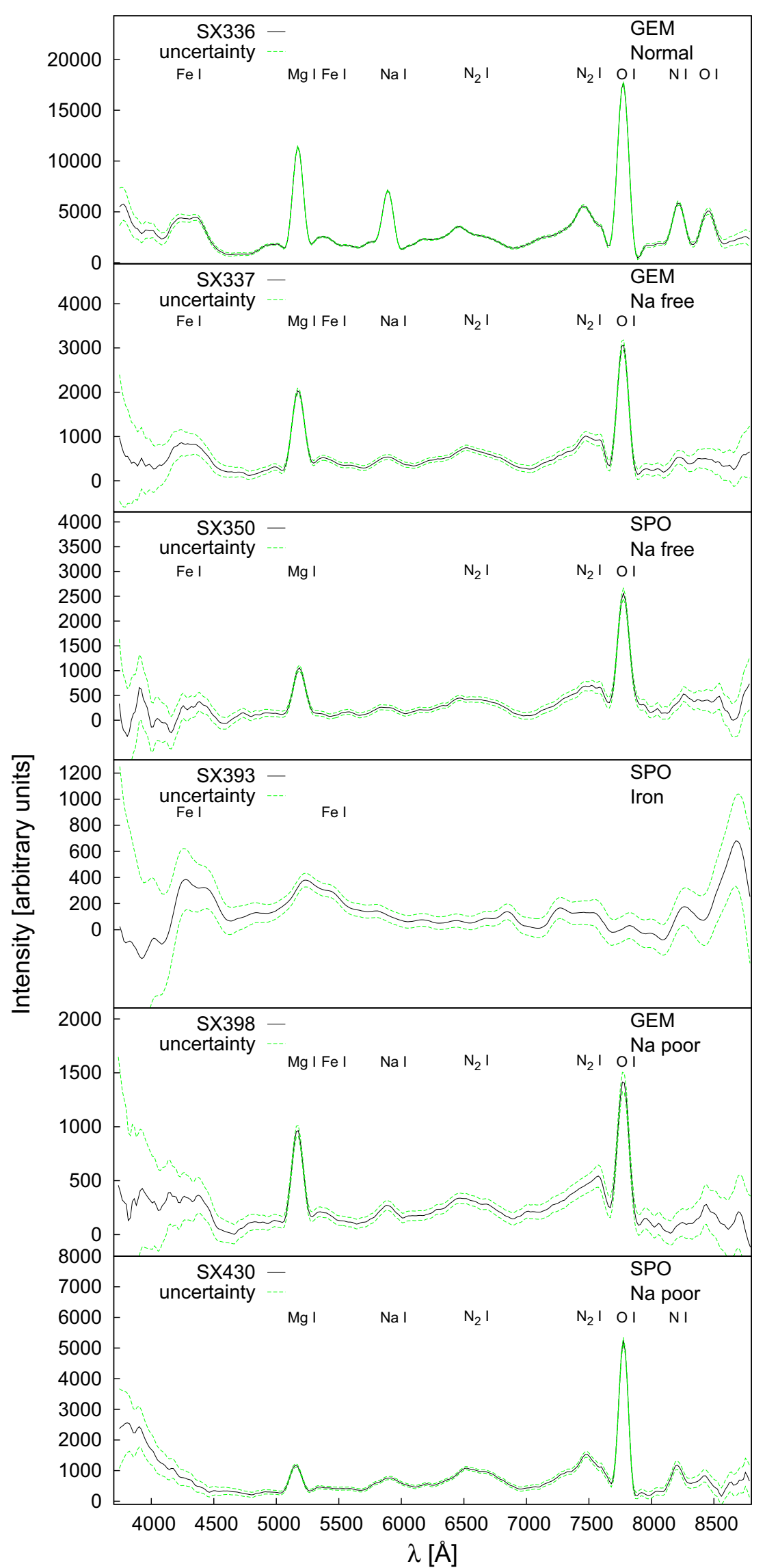

Fig. 20. Individual spectra of the catalogue, their spectral classification and shower identifications (SPO - sporadic meteors, GEM - Geminids). Main lines and emission bands are identified. 
V. Vojáček et al.: Catalogue of representative meteor spectra

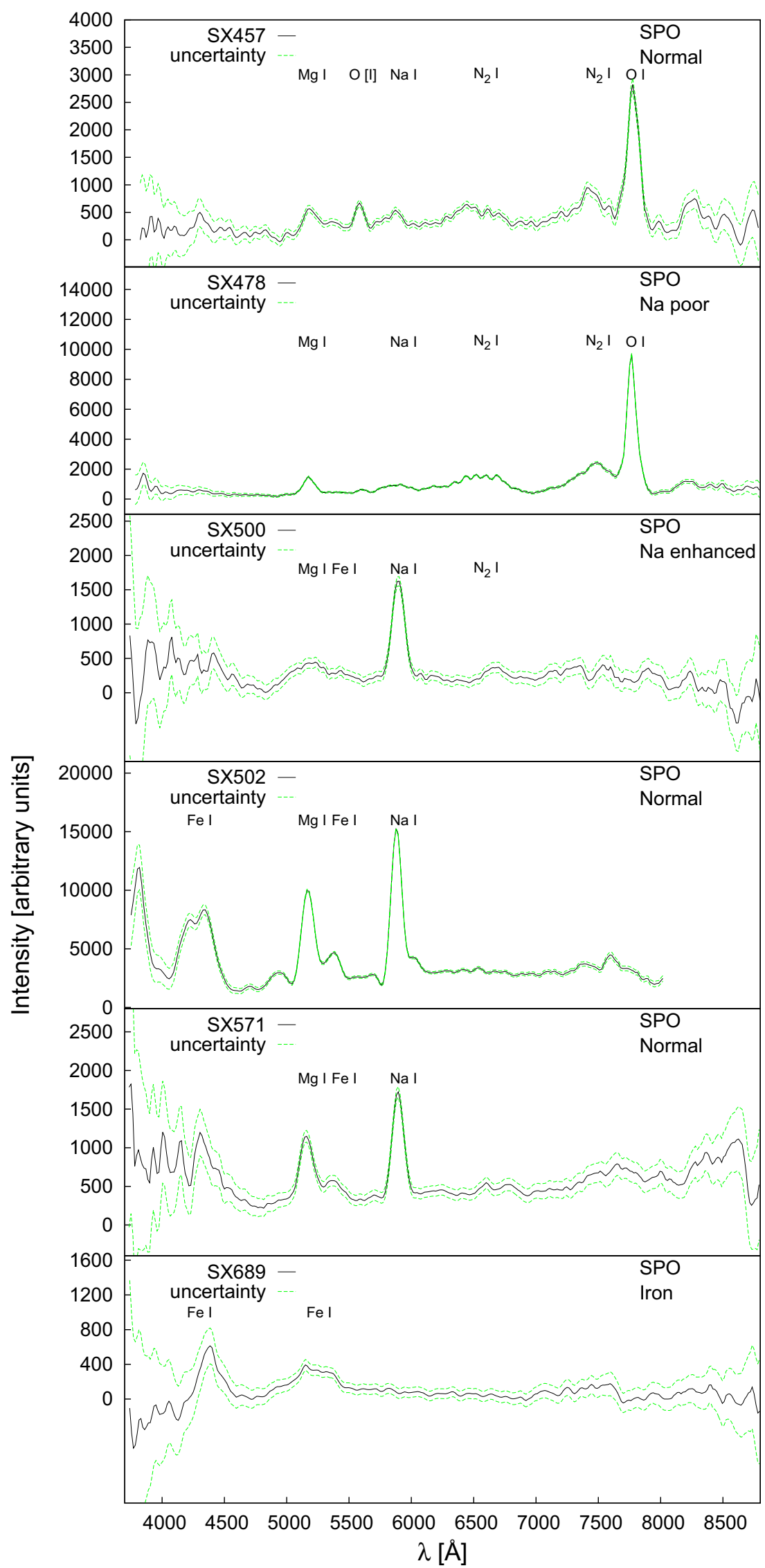

Fig. 21. Individual spectra of the catalogue, their spectral classification and shower identifications (SPO - sporadic meteors, $\sigma$ HYD $-\sigma$ Hydrids. $\kappa \mathrm{CYG}-\kappa$ Cygnids). Main lines and emission bands are identified. 
A\&A 580, A67 (2015)

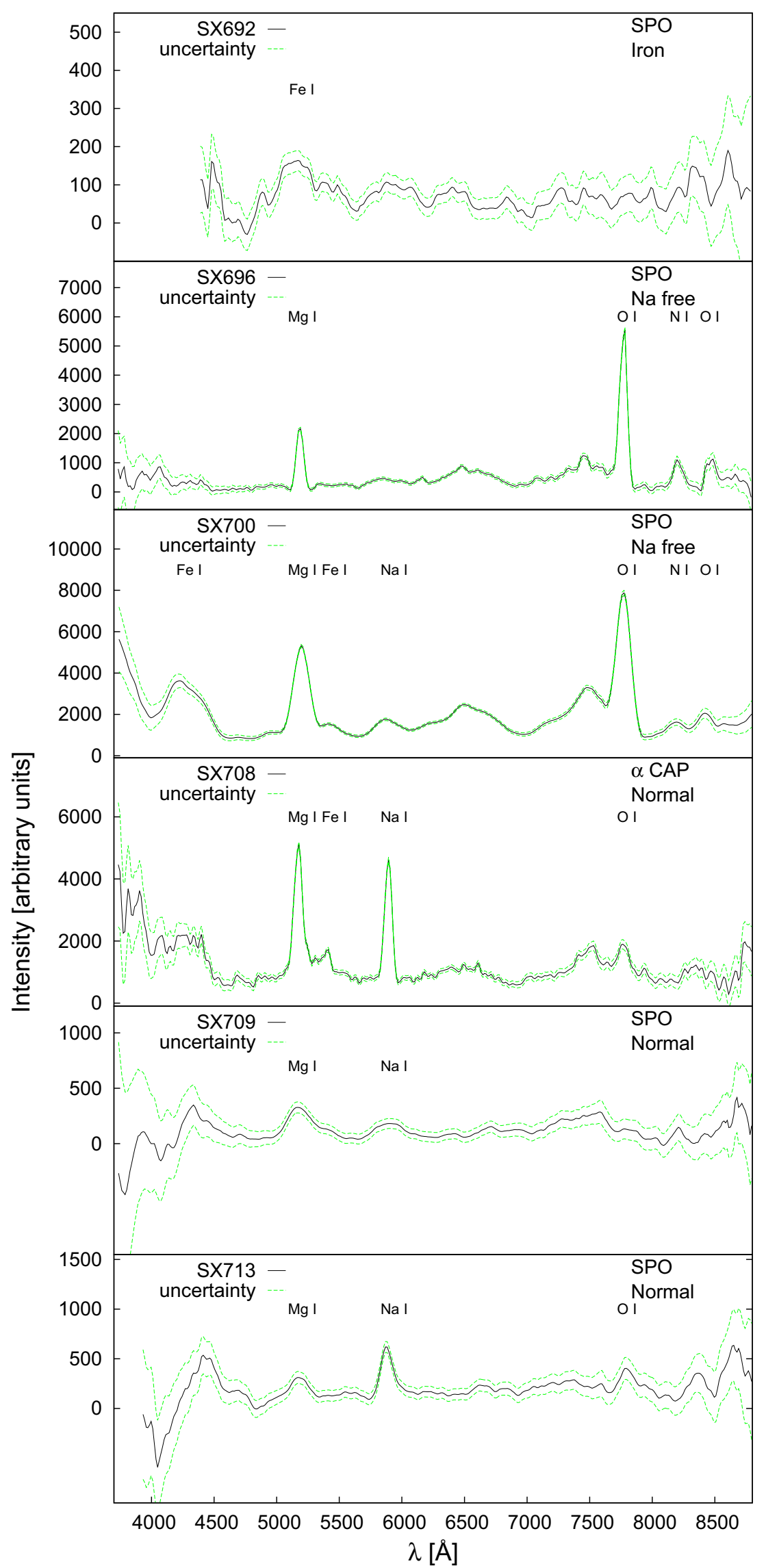

Fig. 22. Individual spectra of the catalogue, their spectral classification and shower identifications (SPO - sporadic meteors, $\alpha$ SCO $-\alpha$ Scorpionids, $\alpha \mathrm{CAP}-\alpha$ Capricornids). Main lines and emission bands are identified. 
V. Vojáček et al.: Catalogue of representative meteor spectra

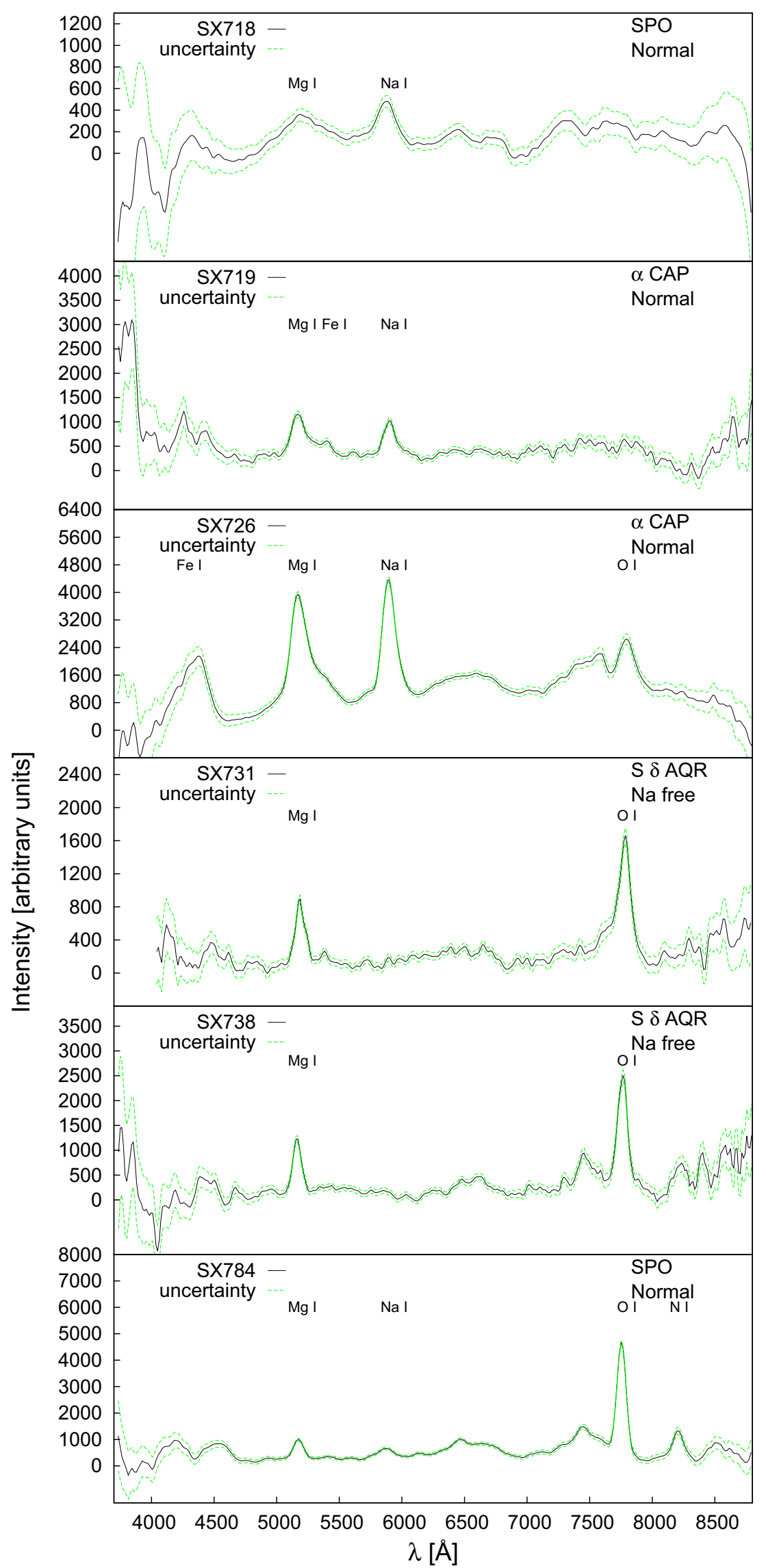

Fig. 23. Individual spectra of the catalogue, their spectral classification and shower identifications (SPO - sporadic meteors, $\alpha$ CAP $\alpha$ Capricornids, $\mathrm{S} \delta \mathrm{AQR}-$ Southern $\delta$ Aquariids). Main lines and emission bands are identified. 
A\&A 580, A67 (2015)

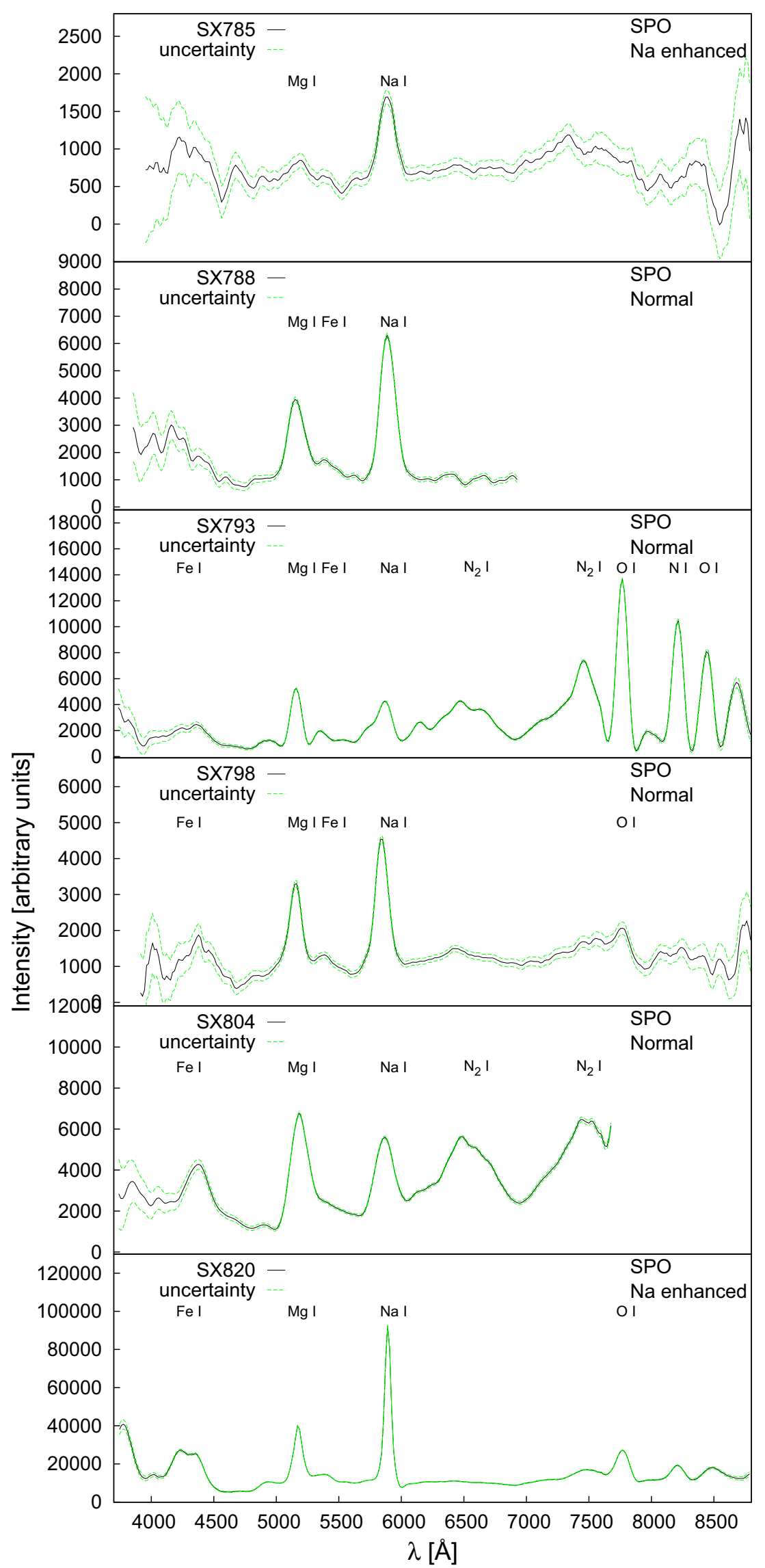

Fig. 24. Individual spectra of the catalogue, their spectral classification and shower identifications (SPO - sporadic meteors, $\kappa$ AQR $-\kappa$ Aquariids, Ann AND - Annual Andromedids). Main lines and emission bands are identified. 
V. Vojáček et al.: Catalogue of representative meteor spectra

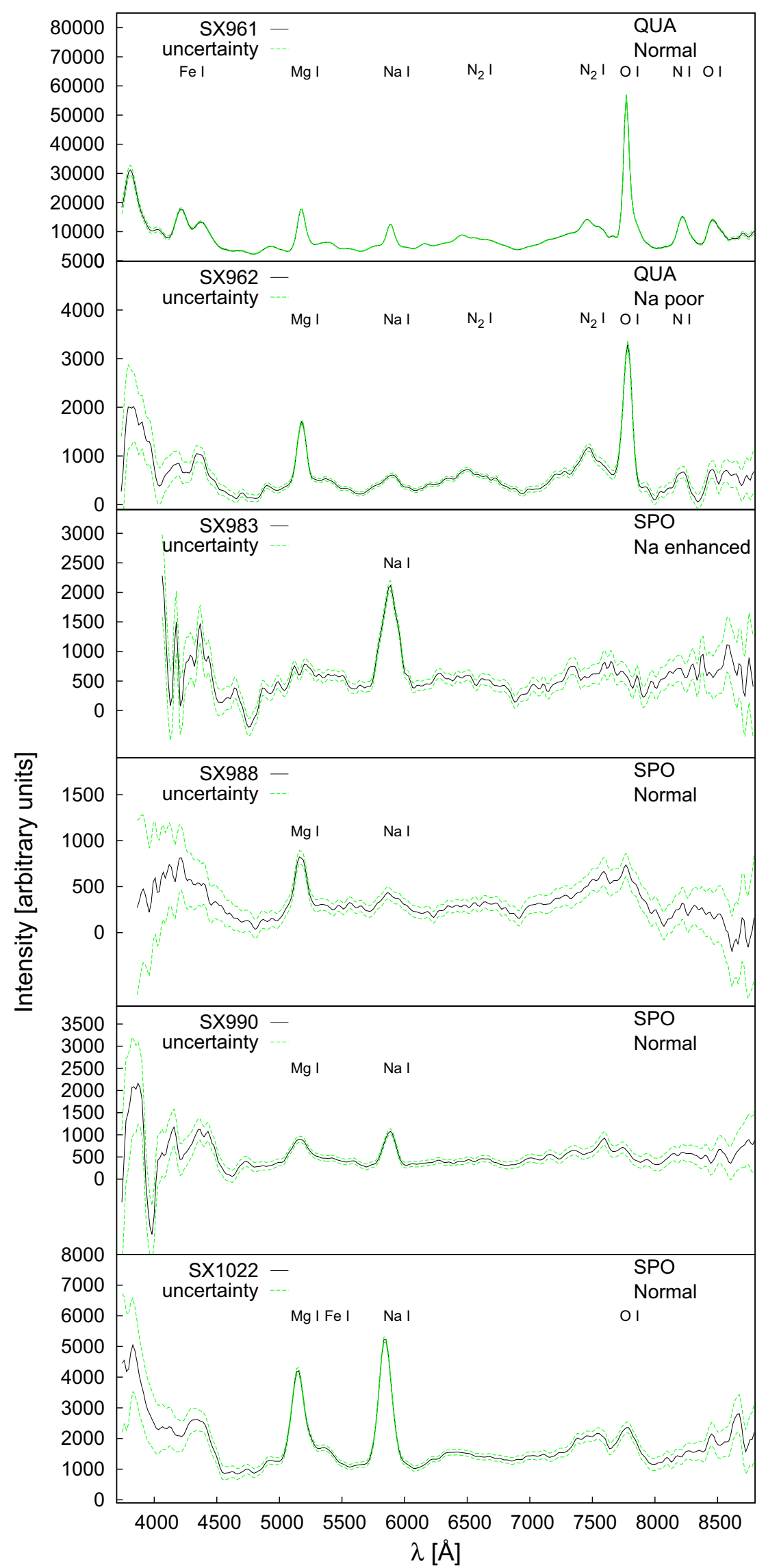

Fig. 25. Individual spectra of the catalogue, their spectral classification and shower identifications (SPO - sporadic meteors, QUA - Quadrantids, $\sigma$ LEO $-\sigma$ Leonids, $\mu$ VIR $-\mu$ Virginids). Main lines and emission bands are identified. 
A\&A 580, A67 (2015)

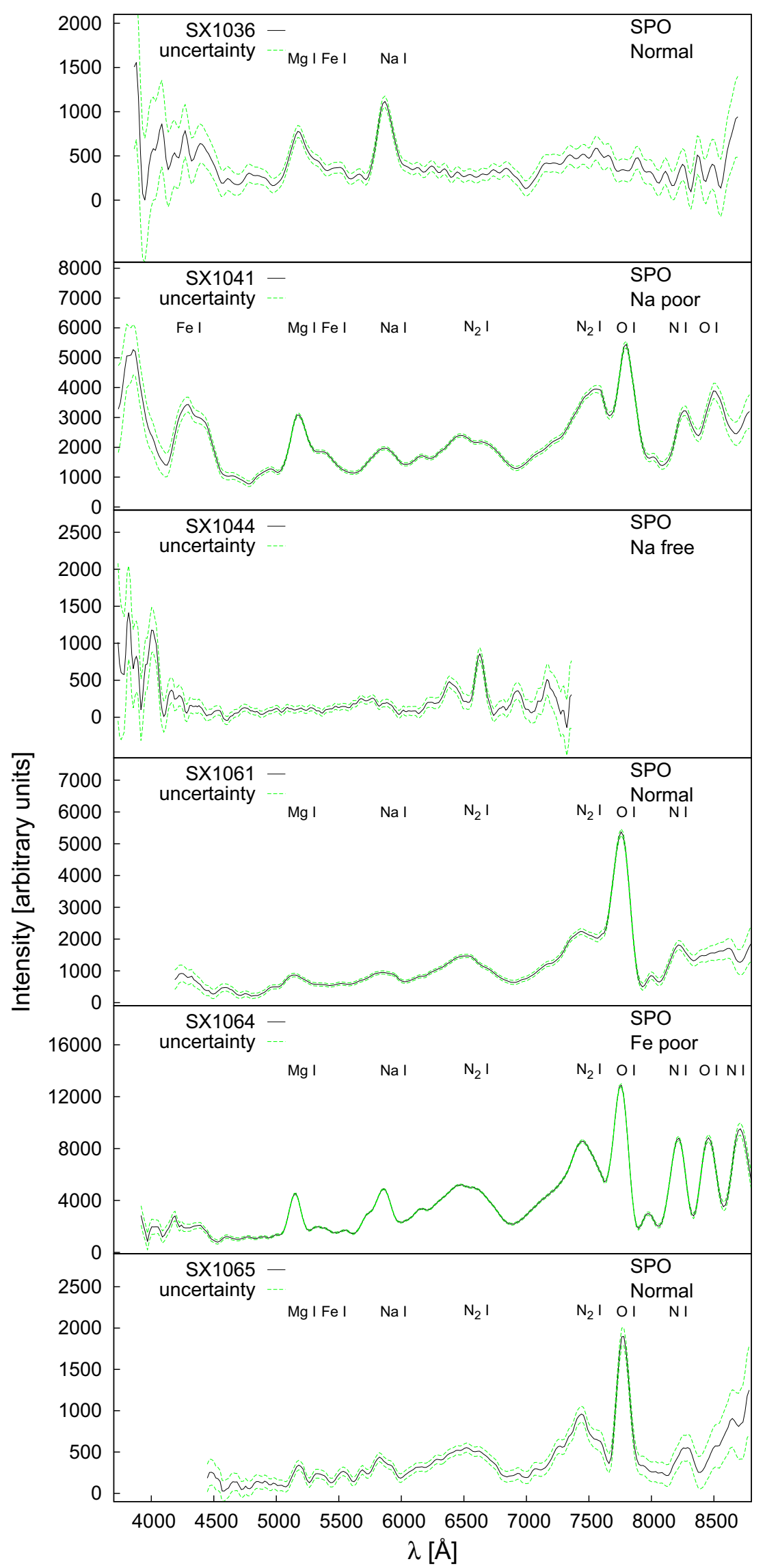

Fig. 26. Individual spectra of the catalogue, their spectral classification and shower identifications (SPO - sporadic meteors, N $\delta$ AQR - Northern $\delta$ Aquariids). Main lines and emission bands are identified. 
V. Vojáček et al.: Catalogue of representative meteor spectra

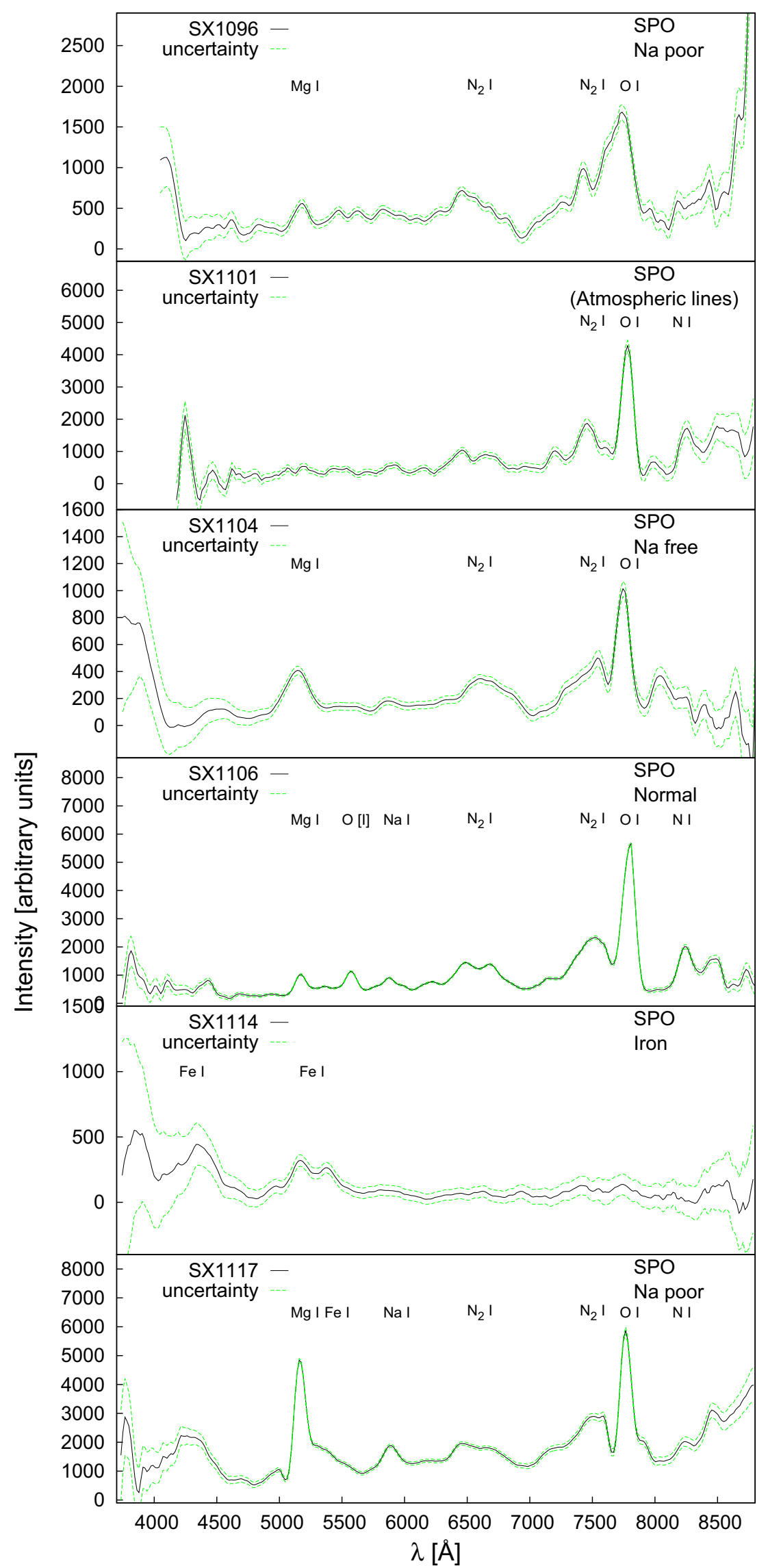

Fig. 27. Individual spectra of the catalogue, their spectral classification and shower identifications (SPO - sporadic meteors). Main lines and emission bands are identified. 
A\&A 580, A67 (2015)

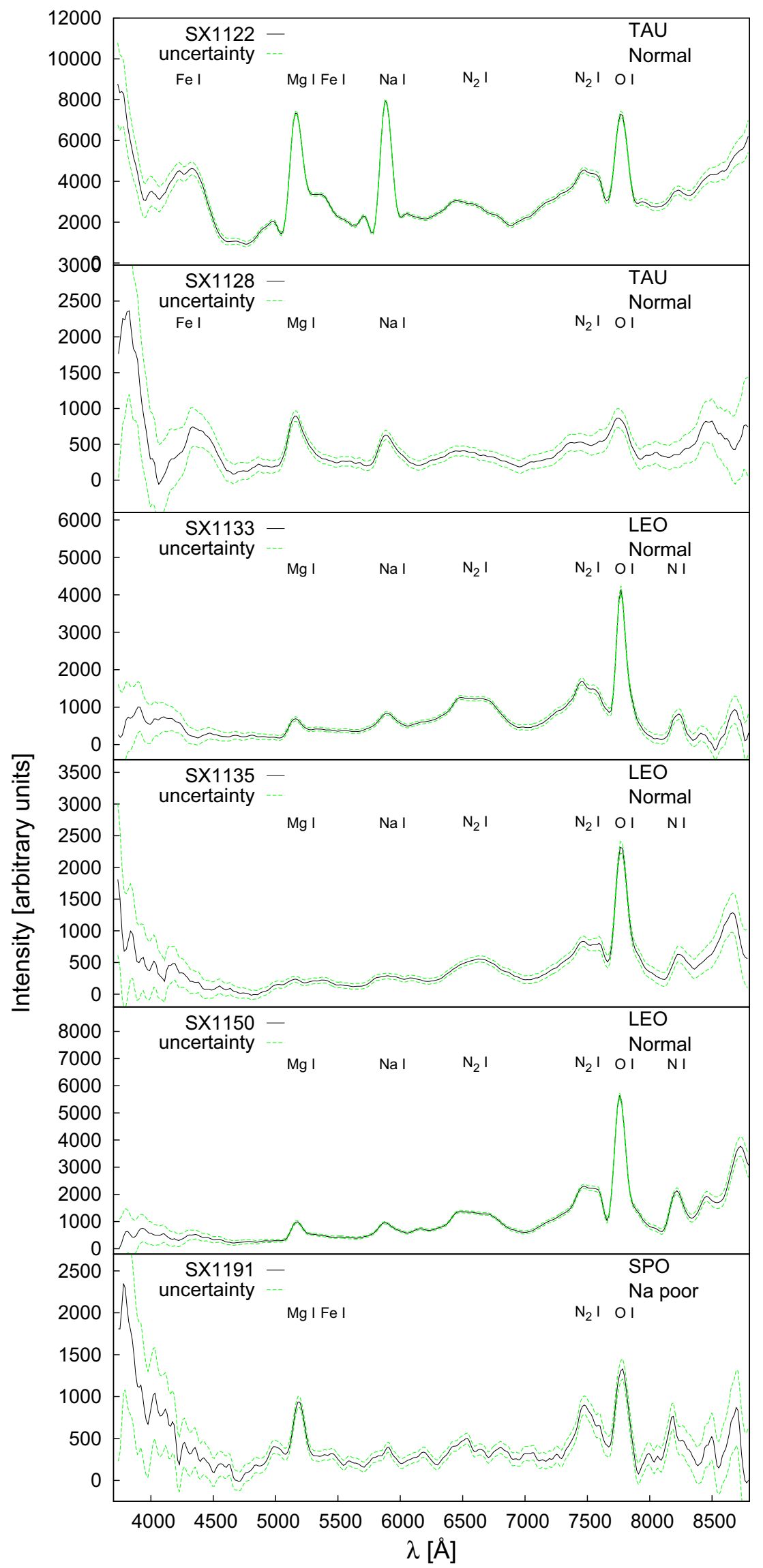

Fig. 28. Individual spectra of the catalogue, their spectral classification and shower identifications (SPO - sporadic meteors, TAU - Taurids, LEO Leonids). Main lines and emission bands are identified. 
V. Vojáček et al.: Catalogue of representative meteor spectra

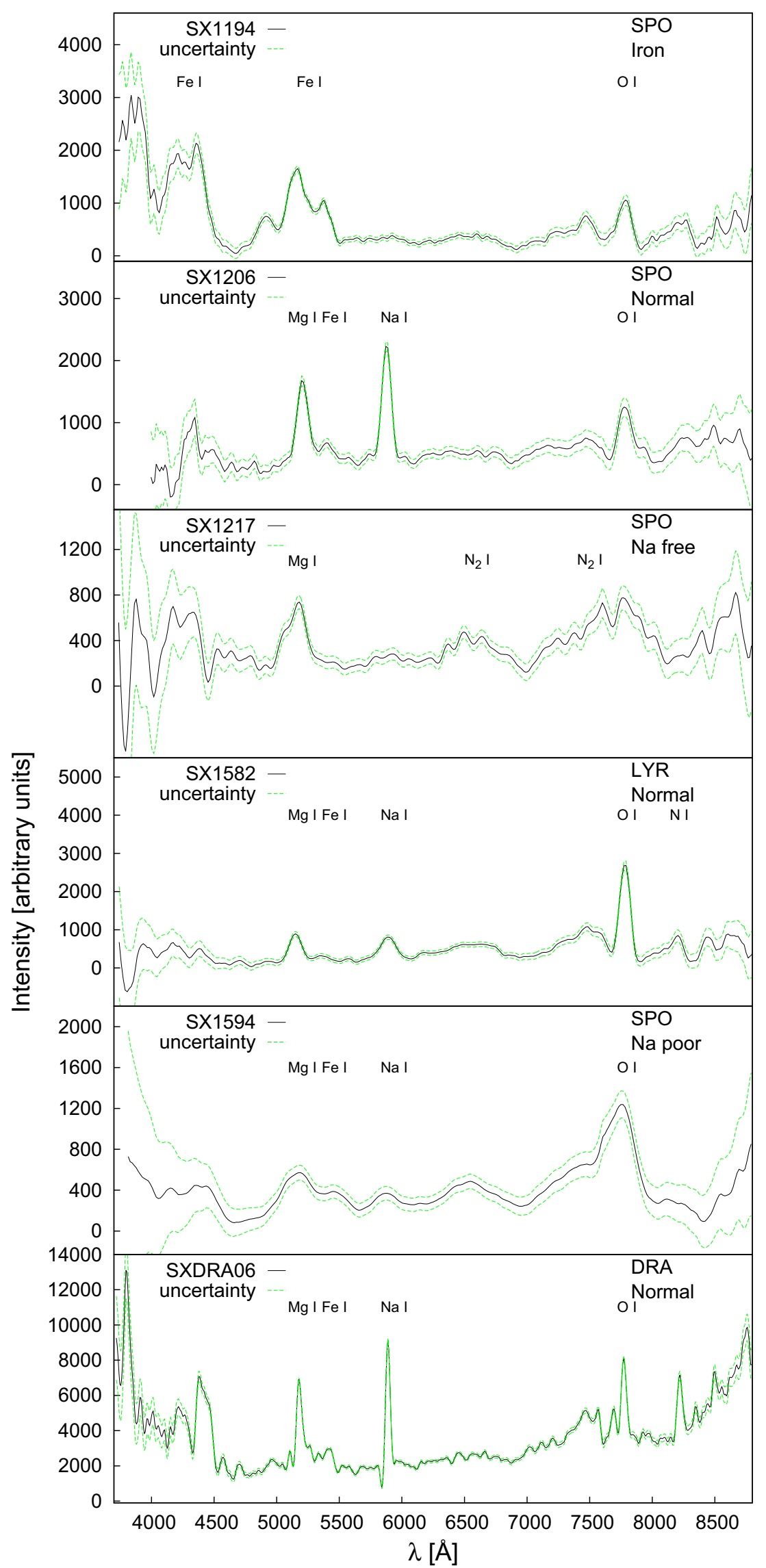

Fig. 29. Individual spectra of the catalogue, their spectral classification and shower identifications (SPO - sporadic meteors, LYR - Lyrids, DRA Draconids). Main lines and emission bands are identified. 
A\&A 580, A67 (2015)

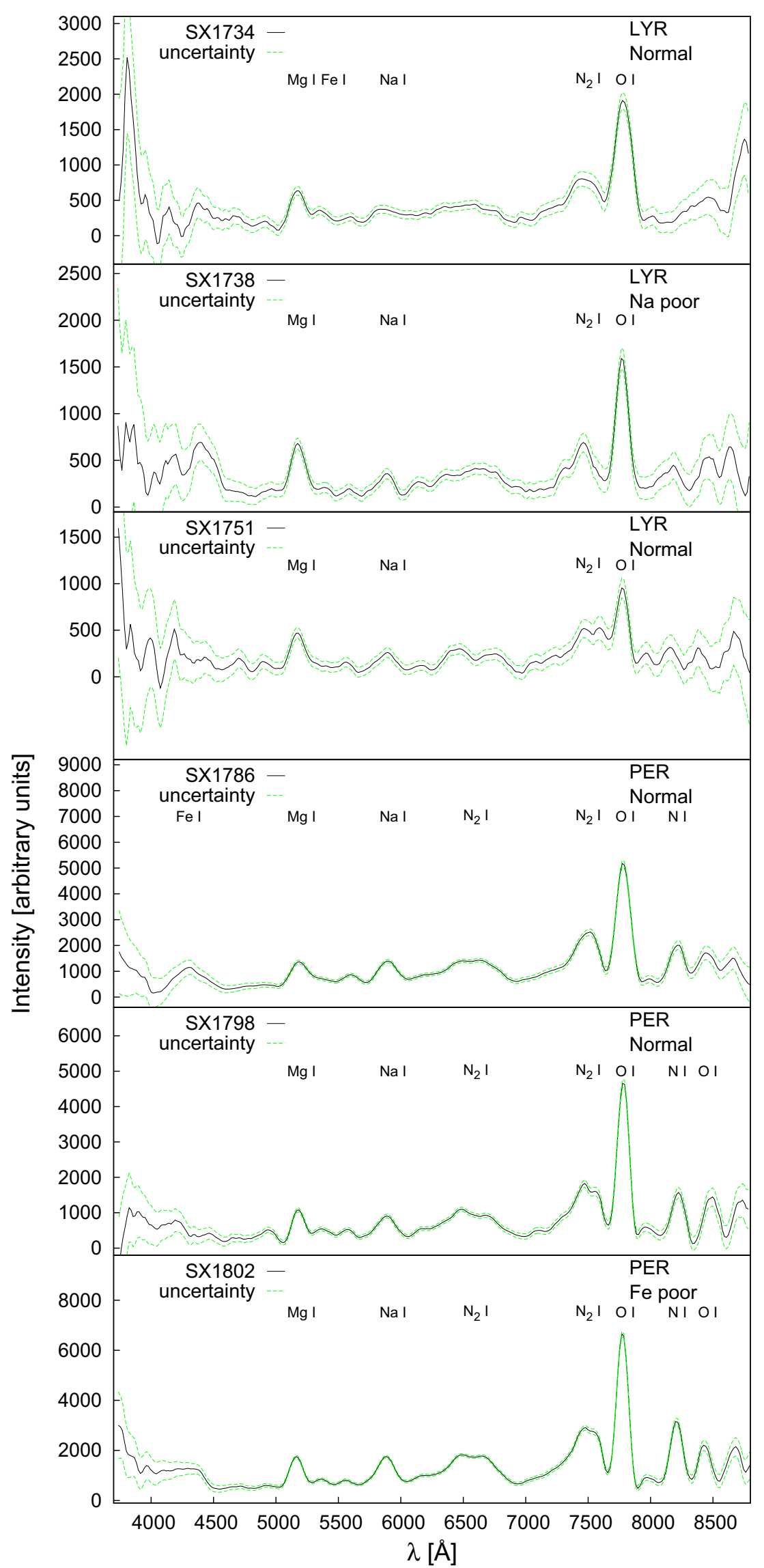

Fig. 30. Individual spectra of the catalogue, their spectral classification and shower identifications (SPO - sporadic meteors, PER - Perseids). Main lines and emission bands are identified. 
Table 4. Atmospheric trajectories of the observed meteors.

\begin{tabular}{|c|c|c|c|c|c|c|c|c|c|c|c|}
\hline Spectrum & Meteor & $\begin{array}{l}\text { Time } \\
\text { (UT) }\end{array}$ & $\begin{array}{l}\alpha_{\mathrm{G}} \\
\left(^{\circ}\right)\end{array}$ & $\begin{array}{l}\delta_{\mathrm{G}} \\
\left({ }^{\circ}\right)\end{array}$ & $\begin{array}{l}z_{\mathrm{R}} \\
\left(^{\circ}\right)\end{array}$ & $\begin{array}{l}H_{\text {beg }} \\
(\mathrm{km})\end{array}$ & $\begin{array}{l}H_{\text {end }} \\
(\mathrm{km})\end{array}$ & $\begin{array}{l}M_{\max } \\
(\mathrm{mag})\end{array}$ & $\begin{array}{l}\text { Photo mass } \\
\quad(\mathrm{g})\end{array}$ & $\begin{array}{c}v \\
\left(\mathrm{~km} \mathrm{~s}^{-1}\right)\end{array}$ & Spectral type \\
\hline \multirow[t]{2}{*}{ SX001 } & 06406048 & $23: 43: 07$ & 223.8 & -6.8 & 57.7 & 98.0 & 84.7 & 0.6 & $2.5 \mathrm{E}-02$ & 41.9 & Iron \\
\hline & & & 0.1 & 0.4 & 0.4 & - & - & - & - & 0.2 & \\
\hline \multirow{2}{*}{ SX002 } & 06406069 & $00: 36: 32$ & 281.28 & 48.81 & 38.85 & 115.7 & 90.6 & 0.5 & $2.2 \mathrm{E}-02$ & 42.2 & Normal \\
\hline & & & 0.09 & 0.05 & 0.06 & - & - & - & - & 0.1 & \\
\hline \multirow[t]{2}{*}{ SX008 } & 06420006 & $20: 35: 16$ & 242.09 & 20.52 & 60.80 & 103.8 & 84.0 & 0.0 & $6.6 \mathrm{E}-02$ & 41.9 & Na-poor \\
\hline & & & 0.05 & 0.04 & 0.04 & - & - & - & - & 0.1 & \\
\hline \multirow[t]{2}{*}{ SX015 } & 06420089 & $00: 40: 41$ & 210.08 & -17.91 & 67.13 & 99.0 & 80.6 & 0.0 & 2.6E-01 & 28.08 & Normal \\
\hline & & & 0.02 & 0.04 & 0.04 & - & - & - & - & 0.09 & \\
\hline \multirow[t]{2}{*}{ SX045 } & 06724023 & $21: 45: 35$ & 323.43 & 3.4 & 55.8 & 103.0 & 84.2 & -0.2 & 7.2E-02 & 37.57 & Na-poor \\
\hline & & & 0.03 & 0.3 & 0.2 & - & - & - & - & 0.09 & \\
\hline \multirow[t]{2}{*}{ SX089 } & 06727196 & $01: 16: 54$ & 276.4 & 35.13 & 44.19 & 98.4 & 79.2 & -0.9 & $5.4 \mathrm{E}-01$ & 22.23 & Normal \\
\hline & & & 0.07 & 0.08 & 0.06 & - & - & - & - & 0.07 & \\
\hline \multirow[t]{2}{*}{ SX090 } & 06727206 & $01: 25: 18$ & 359.66 & 15.64 & 36.66 & 121.7 & 89.4 & -0.8 & 2.2E-02 & 64.4 & Normal \\
\hline & & & 0.09 & 0.07 & 0.07 & - & - & - & - & 0.1 & \\
\hline \multirow[t]{2}{*}{ SX092 } & 06727222 & 01:47:07 & 109.84 & 52.66 & 65.02 & 102.8 & 80.8 & -2.1 & $8.5 \mathrm{E}-01$ & 36.31 & Na-poor \\
\hline & & & 0.04 & 0.02 & 0.02 & - & - & - & - & 0.06 & \\
\hline \multirow[t]{2}{*}{ SX116 } & 06730083 & 23:12:00 & 305.26 & -9.11 & 55.47 & 95.0 & 80.1 & -2.4 & $2.3 \mathrm{E}+00$ & 23.8 & Normal \\
\hline & & & 0.03 & 0.07 & 0.05 & - & - & - & - & 0.1 & \\
\hline \multirow[t]{2}{*}{ SX143 } & 06A20013 & $20: 28: 34$ & 353.78 & 17.22 & 28.84 & 106.6 & 72.0 & -1.4 & $1.2 \mathrm{E}+00$ & 17.60 & Na-enhanced \\
\hline & & & 0.05 & 0.06 & 0.03 & - & - & - & - & 0.08 & \\
\hline \multirow[t]{2}{*}{ SX150 } & 06A20125 & $22: 43: 42$ & 355.2 & 8.3 & 41.8 & 95.2 & 72.6 & 0.7 & $1.5 \mathrm{E}-01$ & 17.30 & Na rich \\
\hline & & & 0.8 & 0.8 & 0.7 & - & - & - & - & 0.05 & \\
\hline \multirow[t]{2}{*}{ SX151 } & 06A20126 & $22: 44: 55$ & 53.7 & 23.6 & 34.2 & 97.1 & 82.1 & 0.9 & $9.0 \mathrm{E}-03$ & 44.4 & Na-free \\
\hline & & & 0.1 & 0.1 & 0.1 & - & - & - & - & 0.4 & \\
\hline \multirow[t]{2}{*}{ SX158 } & 06А20149 & $23: 01: 35$ & 94.67 & 15.32 & 62.57 & 118.4 & 94.7 & 2.0 & 2.3E-03 & 66.8 & Normal \\
\hline & & & 0.08 & 0.07 & 0.07 & - & - & - & - & 0.2 & \\
\hline SX211 & 06А20437 & $02: 10: 43$ & 95.7 & 15.0 & 38.1 & 135.2 & 90.5 & -2.4 & 4.1E-02 & 67.8 & Fe-poor \\
\hline & & & 0.2 & 0.1 & 0.1 & - & - & - & - & 0.3 & \\
\hline SX225 & 06A20527 & $03: 01: 45$ & 95.0 & 15.5 & 34.4 & 120.2 & 87.9 & 1.3 & $3.6 \mathrm{E}-03$ & 68.3 & Normal \\
\hline & & & 0.2 & 0.2 & 0.2 & - & - & - & - & 0.2 & \\
\hline SX237 & 06A20632 & 03:57:45 & 96.0 & 14.5 & 35.2 & 125.6 & 94.2 & 1.0 & $0.7 \mathrm{E}-02$ & 66.2 & Normal \\
\hline & & & 0.3 & 0.3 & 0.3 & - & - & - & - & 0.3 & \\
\hline SX263 & 06B18075 & $02: 18: 34$ & 62.00 & 24.15 & 40.84 & 104.8 & 72.0 & -1.3 & $6.8 \mathrm{E}-01$ & 28.15 & Normal \\
\hline & & & 0.03 & 0.02 & 0.02 & - & - & - & - & 0.09 & \\
\hline SX333 & 06C13104 & $23: 20: 41$ & 113.8 & 32.0 & 26.5 & 102.5 & 76.0 & -1.2 & $2.1 \mathrm{E}-01$ & 36.0 & Na-poor \\
\hline & & & 0.2 & 0.1 & 0.1 & - & - & - & - & 0.1 & \\
\hline SX336 & 06C13136 & $23: 34: 20$ & 113.78 & 32.67 & 23.98 & 104.8 & 67.0 & -1.5 & $5.2 \mathrm{E}-01$ & 35.54 & Normal \\
\hline & & & 0.04 & 0.02 & 0.03 & - & - & - & - & 0.05 & \\
\hline SX337 & $06 \mathrm{C} 13137$ & $23: 34: 36$ & 113.2 & 32.65 & 23.69 & 102.0 & 80.3 & 0.3 & $5.6 \mathrm{E}-02$ & 37.2 & Na-free \\
\hline & & & 0.1 & 0.07 & 0.09 & - & - & - & - & 0.2 & \\
\hline SX350 & 06C13334 & $01: 05: 55$ & 209.80 & 55.04 & 54.20 & 99.9 & 82.1 & 0.3 & 2.3E-02 & 46.1 & Na-free \\
\hline & & & 0.07 & 0.06 & 0.05 & - & - & - & - & 0.3 & \\
\hline SX393 & 06C14187 & $22: 45: 46$ & 359.2 & 40.5 & 39.5 & 78.2 & 69.3 & 2.1 & $2.0 \mathrm{E}-01$ & 12.70 & Iron \\
\hline & & & 0.4 & 0.3 & 0.1 & - & - & - & - & 0.08 & \\
\hline SX398 & 06C14215 & 23:09:09 & 113.4 & 31.45 & 27.64 & 102.1 & 81.0 & -0.3 & $3.8 \mathrm{E}-02$ & 36.9 & Na-poor \\
\hline & & & 0.1 & 0.04 & 0.06 & - & - & - & - & 0.2 & \\
\hline SX430 & $06 \mathrm{C} 14357$ & $01: 25: 38$ & 148.59 & 28.28 & 31.55 & 123.6 & 91.2 & 0.5 & $5.2 \mathrm{E}-03$ & 64.9 & Na-poor \\
\hline & & & 0.07 & 0.04 & 0.05 & - & - & - & - & 0.1 & \\
\hline SX457 & $06 \mathrm{C} 14515$ & 03:19:46 & 129.8 & 2.62 & 48.07 & 114.4 & 93.3 & -1.0 & $3.0 \mathrm{E}-02$ & 59.7 & Normal \\
\hline & & & 0.2 & 0.06 & 0.07 & - & - & - & - & 0.2 & \\
\hline SX478 & $06 \mathrm{C} 14645$ & $04: 52: 24$ & 171.7 & 7.99 & 41.08 & 109.6 & 91.6 & -0.6 & $5.0 \mathrm{E}-03$ & 72.9 & Na-poor \\
\hline & & & 0.1 & 0.06 & 0.06 & - & - & - & - & 0.3 & \\
\hline SX500 & 07407021 & $20: 31: 25$ & 194.70 & 43.65 & 23.74 & 89.3 & 72.0 & 0.1 & 4.5E-01 & 18.2 & Na-enhanced \\
\hline & & & 0.08 & 0.04 & 0.04 & - & - & - & - & 0.1 & \\
\hline SX502 & 07407034 & $21: 40: 46$ & 261 & 85.40 & 36.62 & 86.3 & 67.8 & 0.4 & $2.9 \mathrm{E}-01$ & 19.7 & Normal \\
\hline & & & 1 & 0.06 & 0.08 & - & - & - & - & 0.1 & \\
\hline SX571 & 07812429 & 01:40:39 & 285.7 & 46.8 & 44.70 & 100.7 & 79.7 & -1.5 & $5.8 \mathrm{E}-01$ & 24.7 & Normal \\
\hline & & & 0.1 & 0.1 & 0.08 & - & - & - & - & 0.1 & \\
\hline SX689 & 08505008 & 01:01:14 & 308 & 66.3 & 31.1 & 86.3 & 79.0 & 1.7 & 2.0E-02 & 26.6 & Iron \\
\hline & & & 1 & 0.2 & 0.4 & - & - & - & - & 0.2 & \\
\hline
\end{tabular}

Notes. The table includes the geocentric right ascension $\alpha_{\mathrm{G}}$, declination $\delta_{\mathrm{G}}$, and zenithal distance $z_{\mathrm{R}}$ of the computed radiant, meteor beginning height $H_{\text {beg }}$, terminal height $H_{\text {end }}$, absolute magnitude $M_{\max }$, computed photometric mass, atmospheric velocity of the meteoroid $v$ and finally the classified spectral type of given meteor. 
Table 4. continued.

\begin{tabular}{|c|c|c|c|c|c|c|c|c|c|c|c|}
\hline Spectrum & Meteor & $\begin{array}{l}\text { Time } \\
\text { (UT) }\end{array}$ & $\begin{array}{l}\alpha_{\mathrm{G}} \\
\left(^{\circ}\right)\end{array}$ & $\begin{array}{l}\delta_{\mathrm{G}} \\
\left(^{\circ}\right)\end{array}$ & $\begin{array}{l}z_{\mathrm{R}} \\
\left({ }^{\circ}\right)\end{array}$ & $\begin{array}{l}H_{\text {beg }} \\
(\mathrm{km})\end{array}$ & $\begin{array}{l}H_{\text {end }} \\
(\mathrm{km})\end{array}$ & $\begin{array}{l}M_{\max } \\
(\mathrm{mag})\end{array}$ & $\begin{array}{l}\text { Photo mass } \\
\text { (g) }\end{array}$ & $\begin{array}{c}v \\
\left(\mathrm{~km} \mathrm{~s}^{-1}\right)\end{array}$ & Spectral type \\
\hline \multirow[t]{2}{*}{ SX692 } & 08505025 & $01: 31: 55$ & 203 & 54 & 30 & 78.6 & 75.6 & 0.6 & $1.1 \mathrm{E}-01$ & 16.1 & Iron \\
\hline & & & 3 & 3 & 2 & - & - & - & - & 0.6 & \\
\hline \multirow[t]{2}{*}{ SX696 } & 08506016 & 01:08:38 & 279.0 & 7.3 & 45.6 & 103.7 & 86.5 & -0.7 & $1.8 \mathrm{E}-02$ & 57.4 & Na-free \\
\hline & & & 0.1 & 0.1 & 0.1 & - & - & - & - & 0.4 & \\
\hline \multirow[t]{2}{*}{ SX700 } & 08507002 & $00: 43: 02$ & 246.71 & -22.8 & 70.7 & 97.9 & 82.8 & 0.4 & $5.0 \mathrm{E}-02$ & 38.5 & Na-free \\
\hline & & & 0.09 & 0.2 & 0.2 & - & - & - & - & 0.1 & \\
\hline \multirow[t]{2}{*}{ SX708 } & 08728076 & $22: 21: 19$ & 304.82 & -9.4 & 56.3 & 98.6 & 80.8 & -0.3 & 3.6E-01 & 25.20 & Normal \\
\hline & & & 0.07 & 0.1 & 0.1 & - & - & - & - & 0.08 & \\
\hline \multirow[t]{2}{*}{ SX709 } & 08728078 & $22: 22: 45$ & 279.2 & 51.1 & 11.6 & 99.3 & 83.2 & 1.2 & $4.0 \mathrm{E}-02$ & 29.4 & Normal \\
\hline & & & 0.4 & 0.1 & 0.2 & - & - & - & - & 0.3 & \\
\hline \multirow[t]{2}{*}{ SX713 } & 08728098 & $22: 49: 51$ & 230.0 & 45.8 & 42.7 & 93.6 & 78.3 & 0.8 & 3.2E-01 & 17.41 & Normal \\
\hline & & & 0.3 & 0.2 & 0.2 & - & - & - & - & 0.04 & \\
\hline \multirow[t]{2}{*}{ SX718 } & 08728149 & $23: 32: 27$ & 278.4 & 50.4 & 22.7 & 99.1 & 82.4 & 0.9 & $5.8 \mathrm{E}-02$ & 28.86 & Normal \\
\hline & & & 0.2 & 0.1 & 0.2 & - & - & - & - & 0.08 & \\
\hline \multirow[t]{2}{*}{ SX719 } & 08728151 & $23: 33: 42$ & 304.54 & -9.05 & 56.50 & 101.2 & 82.2 & -0.1 & 2.3E-01 & 24.95 & Normal \\
\hline & & & 0.05 & 0.09 & 0.08 & - & - & - & - & 0.09 & \\
\hline \multirow[t]{2}{*}{ SX726 } & 08728233 & $00: 43: 49$ & 304.46 & -8.67 & 60.30 & 102.3 & 80.4 & -1.0 & 7.4E-01 & 24.80 & Normal \\
\hline & & & 0.03 & 0.05 & 0.04 & - & - & - & - & 0.07 & \\
\hline \multirow[t]{2}{*}{ SX731 } & 08728280 & 01:20:07 & 340.1 & -15.1 & 63.5 & 97.7 & 84.5 & 0.3 & $2.7 \mathrm{E}-02$ & 42.3 & Na-free \\
\hline & & & 0.1 & 0.1 & 0.1 & - & - & - & - & 0.2 & \\
\hline \multirow[t]{2}{*}{ SX738 } & 08729037 & $22: 03: 15$ & 339.53 & -16.46 & 75.64 & 103.1 & 85.4 & 0.0 & 4.2E-02 & 42.5 & Na-free \\
\hline & & & 0.01 & 0.08 & 0.07 & - & - & - & - & 0.1 & \\
\hline SX784 & 08927015 & 23:59:01 & 82.78 & 13.05 & 61.94 & 123.1 & 96.8 & 0.5 & $6.3 \mathrm{E}-03$ & 70.8 & Normal \\
\hline & & & 0.06 & 0.04 & 0.05 & - & - & - & - & 0.1 & \\
\hline SX785 & 08927018 & 00:01:28 & 348.45 & -20.9 & 68.74 & 99.4 & 83.4 & 0.1 & 7.2E-01 & 19.1 & Na-enhanced \\
\hline & & & 0.06 & 0.1 & 0.02 & - & - & - & - & 0.2 & \\
\hline SX788 & 08927101 & 02:01:02 & 309.5 & 37.2 & 62.31 & 96.3 & 76.6 & -0.7 & $1.1 \mathrm{E}+00$ & 19.5 & Normal \\
\hline & & & 0.2 & 0.1 & 0.04 & - & - & - & - & 0.2 & \\
\hline SX793 & 08927195 & $03: 43: 50$ & 134.96 & 52.56 & 35.76 & 118.4 & 86.0 & -2.5 & 9.2E-02 & 60.77 & Normal \\
\hline & & & 0.09 & 0.03 & 0.05 & - & - & - & - & 0.09 & \\
\hline SX798 & 08928139 & $00: 05: 25$ & 2.08 & 10.36 & 40.45 & 101.3 & 74.6 & -0.6 & 7.0E-01 & 24.73 & Normal \\
\hline & & & 0.03 & 0.03 & 0.02 & - & - & - & - & 0.07 & \\
\hline SX804 & 08928235 & $01: 55: 56$ & 203.2 & 85.75 & 43.55 & 114.2 & 86.9 & -1.8 & $1.6 \mathrm{E}-01$ & 42.48 & Normal \\
\hline & & & 0.7 & 0.09 & 0.08 & - & - & - & - & 0.08 & \\
\hline SX820 & 08A20002 & $20: 05: 23$ & 23.78 & 5.59 & 51.77 & 90.0 & 55.8 & -0.7 & $1.1 \mathrm{E}+00$ & 23.98 & Na-enhanced \\
\hline & & & 0.02 & 0.01 & 0.01 & - & - & - & - & 0.03 & \\
\hline SX961 & 09102507 & $05: 28: 21$ & 229.74 & 52.20 & 19.27 & 103.8 & 79.0 & -1.6 & $1.2 \mathrm{E}-01$ & 41.7 & Normal \\
\hline & & & 0.04 & 0.02 & 0.02 & - & - & - & - & 0.2 & \\
\hline SX962 & 09102515 & $05: 31: 10$ & 231.17 & 49.69 & 20.09 & 103.1 & 81.0 & -0.1 & 2.2E-02 & 42.5 & Na-poor \\
\hline & & & 0.07 & 0.03 & 0.04 & - & - & - & - & 0.3 & \\
\hline SX983 & 09421005 & $20: 07: 24$ & 204.4 & 3.28 & 53.25 & 102.9 & 78.2 & -0.1 & $6.4 \mathrm{E}-01$ & 22.40 & Na-enhanced \\
\hline & & & 0.1 & 0.04 & 0.06 & - & - & - & - & 0.02 & \\
\hline SX988 & 09421084 & $00: 14: 38$ & 219.5 & -5.71 & 53.24 & 105.7 & 86.6 & -1.4 & 3.6E-01 & 31.1 & Normal \\
\hline & & & 0.1 & 0.06 & 0.05 & - & - & - & - & 0.2 & \\
\hline SX990 & 09421101 & 00:54:11 & 203.81 & 5.04 & 49.08 & 103.3 & 81.3 & 1.4 & $1.4 \mathrm{E}-01$ & 21.9 & Normal \\
\hline & & & 0.03 & 0.08 & 0.06 & - & - & - & - & 0.1 & \\
\hline SX1022 & 09729261 & 01:16:49 & 297.6 & 3.45 & 55.11 & 103.2 & 75.7 & -1.1 & $1.3 \mathrm{E}+00$ & 23.71 & Normal \\
\hline & & & 0.02 & 0.02 & 0.02 & - & - & - & - & 0.06 & \\
\hline SX1036 & 09818045 & $21: 18: 57$ & 289.34 & 21.35 & 27.19 & 94.5 & 80.4 & 0.8 & 1.7E-01 & 19.37 & Normal \\
\hline & & & 0.06 & 0.09 & 0.08 & - & - & - & - & 0.06 & \\
\hline SX1041 & 09818120 & $23: 23: 17$ & 352.08 & 3.92 & 47.41 & 104.5 & 82.7 & -1.7 & $1.4 \mathrm{E}-01$ & 40.1 & Na-poor \\
\hline & & & 0.07 & 0.09 & 0.09 & - & - & - & - & 0.2 & \\
\hline SX1044 & 09818147 & $23: 58: 43$ & 36.22 & 41.92 & 37.39 & 115.3 & 93.2 & 0.1 & $6.1 \mathrm{E}-03$ & 63.7 & Na-free \\
\hline & & & 0.06 & 0.09 & 0.06 & - & - & - & - & 0.3 & \\
\hline SX1061 & 09819134 & $23: 19: 41$ & 66 & 62.3 & 48.1 & 114.1 & 88.6 & -2.1 & 7.2E-02 & 57.33 & Normal \\
\hline & & & 2 & 0.8 & 0.8 & - & - & - & - & 0.09 & \\
\hline SX1064 & 09819164 & $23: 56: 02$ & 64.0 & 66.65 & 41.70 & 128.0 & 85.0 & -2.8 & $2.0 \mathrm{E}-01$ & 56.2 & Fe-poor \\
\hline & & & 0.1 & 0.03 & 0.04 & - & - & - & - & 0.2 & \\
\hline SX1065 & 09819165 & $23: 56: 50$ & 27.20 & 32.9 & 36.69 & 119.8 & 91.1 & -0.7 & $1.3 \mathrm{E}-02$ & 66.1 & Normal \\
\hline & & & 0.07 & 0.1 & 0.09 & - & - & - & - & 0.5 & \\
\hline SX1096 & 09820151 & $23: 27: 02$ & 21.9 & 28.4 & 40.3 & 116.0 & 92.2 & 0.2 & $5.2 \mathrm{E}-03$ & 60.4 & Na-poor \\
\hline & & & 0.1 & 0.1 & 0.1 & - & - & - & - & 0.2 & \\
\hline SX1101 & 09820190 & $00: 17: 12$ & 53.08 & -10.98 & 81.72 & 119.3 & 99.8 & -0.5 & 4.9E-02 & 64.94 & (Atmospheric lines) \\
\hline & & & 0.01 & 0.05 & 0.04 & - & - & - & - & 0.07 & \\
\hline
\end{tabular}


Table 4. continued.

\begin{tabular}{|c|c|c|c|c|c|c|c|c|c|c|c|}
\hline Spectrum & Meteor & $\begin{array}{l}\text { Time } \\
\text { (UT) }\end{array}$ & $\begin{array}{l}\alpha_{\mathrm{G}} \\
\left({ }^{\circ}\right)\end{array}$ & $\begin{array}{l}\delta_{\mathrm{G}} \\
\left({ }^{\circ}\right)\end{array}$ & $\begin{array}{l}z_{\mathrm{R}} \\
\left({ }^{\circ}\right)\end{array}$ & $\begin{array}{l}H_{\text {beg }} \\
(\mathrm{km})\end{array}$ & $\begin{array}{l}H_{\text {end }} \\
(\mathrm{km})\end{array}$ & $\begin{array}{l}M_{\max } \\
(\mathrm{mag})\end{array}$ & $\begin{array}{l}\text { Photo mass } \\
\text { (g) }\end{array}$ & $\begin{array}{c}v \\
\left(\mathrm{~km} \mathrm{~s}^{-1}\right)\end{array}$ & Spectral type \\
\hline \multirow[t]{2}{*}{ SX1104 } & 09820250 & 01:15:09 & 51.6 & 40.2 & 34.4 & 98.3 & 89.3 & -0.6 & $4.8 \mathrm{E}-03$ & 65 & Na-free \\
\hline & & & 0.4 & 0.8 & 0.5 & - & - & - & - & 1 & \\
\hline \multirow[t]{2}{*}{ SX1106 } & 09820289 & 01:45:07 & 57.60 & 33.10 & 37.89 & 117.6 & 90.4 & -0.9 & $1.4 \mathrm{E}-02$ & 69.4 & Normal \\
\hline & & & 0.05 & 0.04 & 0.04 & - & - & - & - & 0.2 & \\
\hline \multirow[t]{2}{*}{ SX1114 } & 09B17008 & $18: 39: 27$ & 58.7 & 10.9 & 28.6 & 85.1 & 79.2 & 0.8 & 4.0E-02 & 23.8 & Iron \\
\hline & & & 0.5 & 0.3 & 0.3 & - & - & - & - & 0.2 & \\
\hline \multirow{2}{*}{ SX1117 } & 09B17022 & $19: 12: 24$ & 66.38 & 16.81 & 23.64 & 97.0 & 76.0 & -2.5 & $8.6 \mathrm{E}-01$ & 32.0 & Na-poor \\
\hline & & & 0.09 & 0.04 & 0.05 & - & - & - & - & 0.2 & \\
\hline \multirow[t]{2}{*}{ SX1122 } & 09B17055 & $20: 39: 45$ & 63.36 & 14.71 & 23.98 & 107.1 & 71.7 & -1.4 & 8.6E-01 & 27.7 & Normal \\
\hline & & & 0.03 & 0.02 & 0.02 & - & - & - & - & 0.2 & \\
\hline \multirow[t]{2}{*}{ SX1128 } & 09B17084 & $21: 23: 40$ & 62.03 & 25.96 & 22.01 & 100.9 & 78.5 & -0.5 & $1.9 \mathrm{E}-01$ & 29.7 & Normal \\
\hline & & & 0.04 & 0.04 & 0.03 & - & - & - & - & 0.2 & \\
\hline \multirow[t]{2}{*}{ SX1133 } & 09B17115 & $21: 52: 14$ & 154.74 & 21.06 & 55.28 & 117.7 & 93.0 & 1.3 & $2.5 \mathrm{E}-03$ & 72.6 & Normal \\
\hline & & & 0.08 & 0.09 & 0.08 & - & - & - & - & 0.3 & \\
\hline \multirow[t]{2}{*}{ SX1135 } & 09B17123 & 21:59:48 & 154.5 & 22.9 & 52.7 & 121.8 & 94.5 & 1.8 & $1.3 \mathrm{E}-03$ & 72.0 & Normal \\
\hline & & & 0.3 & 0.1 & 0.2 & - & - & - & - & 0.3 & \\
\hline \multirow[t]{2}{*}{ SX1150 } & 09B17192 & 23:03:06 & 153.9 & 22.23 & 40.0 & 123.6 & 92.1 & 0.9 & 2.2E-03 & 71.1 & Normal \\
\hline & & & 0.1 & 0.08 & 0.1 & - & - & - & - & 0.3 & \\
\hline \multirow[t]{2}{*}{ SX1191 } & 10406022 & 21:07:08 & 212.99 & -2.98 & 64.67 & 105.6 & 84.8 & 0.0 & $9.6 \mathrm{E}-02$ & 34.91 & Na-poor \\
\hline & & & 0.05 & 0.03 & 0.04 & - & - & - & - & 0.03 & \\
\hline \multirow[t]{2}{*}{ SX1194 } & 10406060 & $23: 12: 11$ & 207.5 & -8.8 & 57.0 & 92.1 & 80.4 & 0.5 & $4.4 \mathrm{E}-02$ & 32.6 & Iron \\
\hline & & & 0.2 & 0.2 & 0.2 & - & - & - & - & 0.1 & \\
\hline \multirow[t]{2}{*}{ SX1206 } & 10407030 & 21:46:02 & 196.02 & 4.52 & 45.18 & 107.4 & 79.5 & 0.3 & $3.7 \mathrm{E}-01$ & 24.47 & Normal \\
\hline & & & 0.06 & 0.03 & 0.03 & - & - & - & - & 0.06 & \\
\hline \multirow{2}{*}{ SX1217 } & 10408088 & $02: 45: 40$ & 225.3 & -9.9 & 62.5 & 100.2 & 85.6 & -0.1 & $3.8 \mathrm{E}-02$ & 40.6 & Na-free \\
\hline & & & 0.3 & 0.4 & 0.4 & - & - & - & - & 0.3 & \\
\hline \multirow[t]{2}{*}{ SX1582 } & 11422209 & $02: 18: 47$ & 272.7 & 32.90 & 19.17 & 115.3 & 86.4 & -3.1 & 2.7E-01 & 48.5 & Normal \\
\hline & & & 0.2 & 0.05 & 0.08 & - & - & - & - & 0.2 & \\
\hline \multirow[t]{2}{*}{ SX1594 } & 11505072 & 01:05:40 & 245.08 & -16.62 & 65.25 & 109.0 & 89.7 & -1.2 & $1.6 \mathrm{E}-01$ & 42.5 & Na-poor \\
\hline & & & 0.03 & 0.09 & 0.09 & - & - & - & - & 0.1 & \\
\hline \multirow[t]{2}{*}{ SXDRA06 } & DRA06 & $20: 28: 21$ & 262.77 & 55.82 & 39.92 & 105.5 & 76.7 & -3.1 & $1.0 \mathrm{E}+00$ & 23.3 & Normal \\
\hline & & & 0.06 & 0.07 & 0.02 & - & - & - & - & 0.1 & \\
\hline \multirow[t]{2}{*}{ SX1734 } & 12421024 & $20: 41: 33$ & 271.85 & 33.61 & 68.74 & 114.6 & 93.5 & -0.7 & 8.0E-02 & 48.51 & Normal \\
\hline & & & 0.02 & 0.02 & 0.01 & - & - & - & - & 0.03 & \\
\hline \multirow[t]{2}{*}{ SX1738 } & 12421069 & $22: 47: 04$ & 271.68 & 32.68 & 50.27 & 124.7 & 89.4 & -0.7 & 4.4E-02 & 48.9 & Na-poor \\
\hline & & & 0.04 & 0.03 & 0.03 & - & - & - & - & 0.1 & \\
\hline \multirow[t]{2}{*}{ SX1751 } & 12422070 & $00: 23: 19$ & 271.68 & 33.48 & 33.69 & 122.5 & 85.9 & 0.6 & $1.4 \mathrm{E}-02$ & 48.32 & Normal \\
\hline & & & 0.05 & 0.02 & 0.04 & - & - & - & - & 0.06 & \\
\hline \multirow[t]{2}{*}{ SX1786 } & 12811093 & $22: 27: 52$ & 46.82 & 57.77 & 52.61 & 131.3 & 87.2 & -2.0 & 8.3E-02 & 60.87 & Normal \\
\hline & & & 0.07 & 0.04 & 0.04 & - & - & - & - & 0.06 & \\
\hline \multirow[t]{2}{*}{ SX1798 } & 12811305 & 00:29:01 & 48.29 & 56.91 & 38.93 & 129.7 & 90.6 & -0.2 & $2.0 \mathrm{E}-02$ & 61.59 & Normal \\
\hline & & & 0.09 & 0.04 & 0.05 & - & - & - & - & 0.08 & \\
\hline SX1802 & 12811384 & 01:07:25 & 46.09 & 56.74 & 32.89 & 136.7 & 87.7 & -1.6 & 4.3E-02 & 61.1 & $\mathrm{Fe}$-poor \\
\hline & & & 0.09 & 0.02 & 0.05 & - & - & - & - & 0.2 & \\
\hline
\end{tabular}

Table 5. Orbital parameters of observed meteors.

\begin{tabular}{ccccccccccc}
\hline \hline Spectrum & $\begin{array}{c}1 / a \\
(1 / \mathrm{AU})\end{array}$ & $e$ & $\begin{array}{c}i \\
\left(^{\circ}\right)\end{array}$ & $\begin{array}{c}q \\
(\mathrm{AU})\end{array}$ & $\begin{array}{c}Q \\
(\mathrm{AU})\end{array}$ & $\begin{array}{c}\omega \\
\left({ }^{\circ}\right)\end{array}$ & $\Omega$ & $\begin{array}{c}T_{j} \\
\left({ }^{\circ}\right)\end{array}$ & $\begin{array}{c}v_{\mathrm{g}} \\
\left(\mathrm{km} \mathrm{s}^{-1}\right)\end{array}$ & Shower \\
\hline SX001 & 0.395 & 0.957 & 28.9 & 0.110 & 5.0 & 325.1 & 16.95 & 2.4 & 40.3 & SPO \\
& 0.016 & 0.002 & 0.9 & 0.004 & 0.2 & 0.7 & - & 0.3 & 0.2 & \\
SX002 & 0.013 & 0.99 & 66.3 & 0.999 & 160 & 174.5 & 16.98 & 0.6 & 40.5 & SPO \\
& 0.010 & 0.01 & 0.1 & 0.001 & 130 & 0.1 & - & 0.3 & 0.1 & \\
SX008 & 0.047 & 0.974 & 56.7 & 0.563 & 42 & 263.7 & 30.54 & 0.7 & 40.2 & SPO \\
& 0.010 & 0.005 & 0.2 & 0.001 & 9 & 0.2 & - & 0.1 & 0.1 & \\
SX015 & 0.377 & 0.812 & 4.88 & 0.498 & 4.81 & 97.21 & 210.70 & 2.79 & 25.9 & SPO \\
& 0.005 & 0.003 & 0.03 & 0.001 & 0.08 & 0.04 & - & 0.03 & 0.1 & \\
SX045 & 0.512 & 0.898 & 35.0 & 0.200 & 3.71 & 314.2 & 121.76 & 3.1 & 35.7 & SPO \\
& 0.006 & 0.002 & 0.5 & 0.002 & 0.05 & 0.2 & - & 0.2 & 0.1 & \\
SX089 & 0.283 & 0.733 & 26.3 & 0.943 & 6.1 & 213.73 & 124.77 & 2.48 & 19.50 & SPO \\
& 0.005 & 0.004 & 0.1 & 0.001 & 0.1 & 0.09 & - & 0.06 & 0.09 & \\
\hline
\end{tabular}

Notes. Second row for each meteor contains corresponding errors. The standard error for the ascending node $\Omega$ is of order $10^{-3}$ for all meteoroids. 
Table 5. continued.

\begin{tabular}{|c|c|c|c|c|c|c|c|c|c|c|}
\hline Spectrum & $\begin{array}{c}1 / a \\
(1 / \mathrm{AU})\end{array}$ & $\bar{e}$ & $\begin{array}{c}i \\
\left(^{\circ}\right)\end{array}$ & $\begin{array}{c}q \\
(\mathrm{AU})\end{array}$ & $\begin{array}{c}Q \\
(\mathrm{AU})\end{array}$ & $\begin{array}{l}\omega \\
\left(^{\circ}\right)\end{array}$ & $\overline{\bar{\Omega} \Omega}$ & $\begin{array}{l}T_{j} \\
\left({ }^{\circ}\right)\end{array}$ & $\begin{array}{c}v_{\mathrm{g}} \\
\left(\mathrm{km} \mathrm{s}^{-1}\right)\end{array}$ & Shower \\
\hline \multirow[t]{2}{*}{ SX090 } & 0.023 & 0.989 & 147.1 & 0.495 & 86 & 271.8 & 124.78 & -0.6 & 63.3 & SPO \\
\hline & 0.013 & 0.006 & 0.2 & 0.003 & 50 & 0.5 & - & 0.3 & 0.2 & \\
\hline \multirow[t]{2}{*}{ SX092 } & 0.383 & 0.844 & 42.80 & 0.408 & 4.82 & 72.13 & 124.79 & 2.55 & 34.40 & SPO \\
\hline & 0.003 & 0.001 & 0.09 & 0.001 & 0.05 & 0.08 & - & 0.05 & 0.06 & \\
\hline \multirow[t]{2}{*}{ SX116 } & 0.436 & 0.730 & 7.00 & 0.619 & 3.97 & 265.72 & 127.56 & 3.17 & 21.1 & $\alpha \mathrm{CAP}$ \\
\hline & 0.007 & 0.005 & 0.08 & 0.002 & 0.07 & 0.06 & - & 0.04 & 0.1 & \\
\hline \multirow[t]{2}{*}{ SX143 } & 0.396 & 0.657 & 6.84 & 0.865 & 4.19 & 227.95 & 207.21 & 3.10 & 13.6 & SPO \\
\hline & 0.006 & 0.005 & 0.06 & 0.001 & 0.07 & 0.06 & - & 0.04 & 0.1 & \\
\hline \multirow[t]{2}{*}{ SX150 } & 0.358 & 0.69 & 3.5 & 0.872 & 4.7 & 225.9 & 207.30 & 2.92 & 13.43 & SPO \\
\hline & 0.012 & 0.01 & 0.3 & 0.004 & 0.2 & 0.9 & - & 0.08 & 0.06 & \\
\hline \multirow{2}{*}{ SX151 } & 0.316 & 0.985 & 19.9 & 0.048 & 6.3 & 336.6 & 207.31 & 1.9 & 42.9 & SPO \\
\hline & 0.028 & 0.002 & 0.8 & 0.002 & 0.6 & 0.4 & - & 0.2 & 0.4 & \\
\hline \multirow[t]{2}{*}{ SX158 } & 0.161 & 0.908 & 163.0 & 0.576 & 12 & 83.5 & 27.32 & 0.0 & 65.6 & ORI \\
\hline & 0.015 & 0.008 & 0.1 & 0.004 & 1 & 0.7 & - & 0.1 & 0.2 & \\
\hline \multirow{2}{*}{ SX211 } & 0.083 & 0.95 & 162.8 & 0.619 & 23 & 77 & 27.45 & -0.5 & 66.8 & ORI \\
\hline & 0.024 & 0.02 & 0.3 & 0.006 & 7 & 1 & - & 0.3 & 0.3 & \\
\hline \multirow{2}{*}{ SX225 } & 0.015 & 0.99 & 163.9 & 0.610 & 130 & 77.2 & 27.49 & -1 & 67.3 & ORI \\
\hline & 0.022 & 0.01 & 0.4 & 0.005 & 200 & 0.9 & - & 1 & 0.2 & \\
\hline \multirow[t]{2}{*}{ SX237 } & 0.211 & 0.87 & 161.7 & 0.598 & 9 & 82 & 27.53 & 0.2 & 65.3 & ORI \\
\hline & 0.023 & 0.01 & 0.5 & 0.008 & 1 & 1 & - & 0.2 & 0.3 & \\
\hline \multirow[t]{2}{*}{ SX263 } & 0.461 & 0.802 & 3.09 & 0.430 & 3.91 & 285.67 & 236.50 & 3.17 & 26.1 & TAU \\
\hline & 0.005 & 0.003 & 0.03 & 0.001 & 0.05 & 0.06 & - & 0.02 & 0.1 & \\
\hline SX333 & 0.773 & 0.894 & 23.1 & 0.137 & 2.46 & 325.4 & 261.72 & 4.43 & 34.1 & GEM \\
\hline & 0.009 & 0.002 & 0.3 & 0.002 & 0.03 & 0.2 & - & 0.07 & 0.2 & \\
\hline SX336 & 0.751 & 0.895 & 24.8 & 0.139 & 2.52 & 324.62 & 261.73 & 4.51 & 34.5 & GEM \\
\hline & 0.006 & 0.001 & 0.2 & 0.001 & 0.02 & 0.06 & - & 0.02 & 0.1 & \\
\hline SX337 & 0.691 & 0.906 & 25.4 & 0.136 & 2.76 & 324.4 & 261.73 & 4.00 & 35.4 & GEM \\
\hline & 0.009 & 0.002 & 0.3 & 0.001 & 0.04 & 0.2 & - & 0.07 & 0.2 & \\
\hline SX350 & 0.289 & 0.72 & 78.4 & 0.984 & 5.9 & 179.5 & 261.79 & 1.7 & 44.5 & SPO \\
\hline & 0.019 & 0.02 & 0.3 & 0.001 & 0.5 & 0.2 & - & 0.3 & 0.3 & \\
\hline SX393 & 0.631 & 0.384 & 6.4 & 0.976 & 2.19 & 194.1 & 262.71 & 4.3 & 6.5 & SPO \\
\hline & 0.009 & 0.009 & 0.2 & 0.001 & 0.05 & 0.3 & - & 0.2 & 0.2 & \\
\hline SX398 & 0.688 & 0.905 & 22.1 & 0.139 & 2.77 & 324.0 & 262.73 & 4.00 & 35.0 & GEM \\
\hline & 0.013 & 0.003 & 0.3 & 0.002 & 0.06 & 0.2 & - & 0.08 & 0.3 & \\
\hline SX430 & 0.017 & 0.993 & 144.7 & 0.425 & 120 & 278.1 & 278.1 & -0.6 & 63.8 & SPO \\
\hline & 0.013 & 0.006 & 0.1 & 0.003 & 100 & 0.5 & - & 0.4 & 0.2 & \\
\hline SX457 & 0.042 & 0.991 & 129.7 & 0.222 & 50 & 123.8 & 81.89 & -0.1 & 58.7 & SPO \\
\hline & 0.021 & 0.005 & 0.4 & 0.003 & 20 & 0.7 & - & 0.3 & 0.2 & \\
\hline SX478 & 0.049 & 0.95 & 173.0 & 0.977 & 40 & 190.2 & 261.96 & -1.0 & 72.0 & SPO \\
\hline & 0.025 & 0.02 & 0.1 & 0.001 & 20 & 0.4 & - & 0.6 & 0.3 & \\
\hline SX500 & 0.484 & 0.553 & 16.4 & 0.924 & 3.21 & 218.42 & 17.55 & 3.52 & 14.3 & SPO \\
\hline & 0.008 & 0.008 & 0.1 & 0.001 & 0.07 & 0.09 & - & 0.09 & 0.1 & \\
\hline SX502 & 0.360 & 0.641 & 23.8 & 0.998 & 4.6 & 172.7 & 17.59 & 2.90 & 16.3 & SPO \\
\hline & 0.008 & 0.008 & 0.2 & 0.001 & 0.1 & 0.1 & - & 0.09 & 0.1 & \\
\hline SX571 & 0.256 & 0.753 & 32.9 & 0.964 & 6.8 & 207.6 & 139.86 & 2.3 & 22.3 & SPO \\
\hline & 0.007 & 0.007 & 0.2 & 0.001 & 0.2 & 0.1 & - & 0.1 & 0.1 & \\
\hline SX689 & 0.542 & 0.48 & 41.3 & 0.966 & 2.72 & 150 & 45.77 & 3.6 & 24.1 & SPO \\
\hline & 0.011 & 0.01 & 0.3 & 0.002 & 0.08 & 1 & - & 0.2 & 0.2 & \\
\hline SX692 & 0.477 & 0.52 & 16 & 0.999 & 3.2 & 194 & 45.80 & 3.5 & 11.8 & SPO \\
\hline & 0.044 & 0.05 & 1 & 0.004 & 0.4 & 3 & - & 0.6 & 0.8 & \\
\hline SX696 & 0.066 & 0.97 & 108.4 & 0.524 & 30 & 269 & 46.75 & 0.1 & 56.2 & SPO \\
\hline & 0.033 & 0.02 & 0.4 & 0.006 & 20 & 1 & - & 0.4 & 0.4 & \\
\hline SX700 & 0.374 & 0.941 & 2.4 & 0.159 & 5.2 & 137.7 & 227.68 & 2.43 & 36.9 & SPO \\
\hline & 0.007 & 0.001 & 0.4 & 0.001 & 0.1 & 0.2 & - & 0.03 & 0.1 & \\
\hline SX708 & 0.385 & 0.772 & 7.48 & 0.592 & 4.60 & 267.5 & 126.08 & 2.89 & 22.58 & $\alpha$ CAP \\
\hline & 0.005 & 0.003 & 0.09 & 0.001 & 0.07 & 0.1 & - & 0.03 & 0.09 & \\
\hline SX709 & 0.026 & 0.97 & 40.0 & 0.982 & 70 & 201.2 & 126.07 & 1.1 & 27.3 & SPO \\
\hline & 0.023 & 0.02 & 0.3 & 0.001 & 70 & 0.3 & - & 0.6 & 0.3 & \\
\hline SX713 & 0.355 & 0.640 & 18.30 & 1.014 & 4.63 & 175.5 & 126.09 & 2.92 & 13.65 & SPO \\
\hline & 0.004 & 0.004 & 0.08 & 0.001 & 0.06 & 0.2 & - & 0.04 & 0.05 & \\
\hline SX718 & 0.038 & 0.96 & 39.0 & 0.981 & 50 & 201.3 & 126.12 & 1.1 & 26.76 & SPO \\
\hline & 0.007 & 0.07 & 0.1 & 0.001 & 10 & 0.2 & - & 0.1 & 0.08 & \\
\hline SX719 & 0.388 & 0.768 & 7.65 & 0.598 & 4.56 & 266.9 & 126.12 & 2.91 & 22.4 & $\alpha \mathrm{CAP}$ \\
\hline & 0.005 & 0.003 & 0.08 & 0.001 & 0.07 & 0.1 & - & 0.03 & 0.1 & \\
\hline
\end{tabular}


Table 5. continued.

\begin{tabular}{|c|c|c|c|c|c|c|c|c|c|c|}
\hline Spectrum & $\begin{array}{c}1 / a \\
(1 / \mathrm{AU})\end{array}$ & $\bar{e}$ & $\begin{array}{c}i \\
\left({ }^{\circ}\right) \\
\end{array}$ & $\begin{array}{c}q \\
(\mathrm{AU})\end{array}$ & $\begin{array}{c} \\
(\mathrm{AU}) \\
\end{array}$ & $\begin{array}{l}\omega \\
\left({ }^{\circ}\right) \\
\end{array}$ & $\bar{\Omega}$ & $\begin{array}{l}T_{j} \\
\left({ }^{\circ}\right) \\
\end{array}$ & $\begin{array}{c}\begin{array}{c}v_{\mathrm{g}} \\
\left(\mathrm{km} \mathrm{s}^{-1}\right)\end{array} \\
\end{array}$ & Shower \\
\hline \multirow[t]{2}{*}{ SX726 } & 0.391 & 0.766 & 7.91 & 0.600 & 4.52 & 266.78 & 126.17 & 2.93 & 22.33 & $\alpha \mathrm{CAP}$ \\
\hline & 0.004 & 0.003 & 0.05 & 0.001 & 0.05 & 0.07 & - & 0.02 & 0.08 & \\
\hline \multirow[t]{2}{*}{ SX731 } & 0.439 & 0.973 & 25.7 & 0.061 & 4.5 & 154.9 & 306.19 & 2.56 & 40.8 & $\mathrm{~S} \delta \mathrm{AQR}$ \\
\hline & 0.012 & 0.001 & 0.6 & 0.001 & 0.1 & 0.3 & - & 0.09 & 0.2 & \\
\hline \multirow[t]{2}{*}{ SX738 } & 0.363 & 0.971 & 26.3 & 0.080 & 5.4 & 150.5 & 307.01 & 2.20 & 40.8 & $\mathrm{~S} \delta \mathrm{AQR}$ \\
\hline & 0.009 & 0.001 & 0.3 & 0.001 & 0.1 & 0.1 & - & 0.06 & 0.1 & \\
\hline \multirow[t]{2}{*}{ SX784 } & 0.018 & 0.98 & 161.78 & 0.891 & 110 & 39.1 & 4.14 & -1.0 & 69.7 & SPO \\
\hline & 0.011 & 0.01 & 0.08 & 0.001 & 70 & 0.3 & - & 0.5 & 0.1 & \\
\hline \multirow[t]{2}{*}{ SX785 } & 0.276 & 0.766 & 6.26 & 0.848 & 6.4 & 49.8 & 6.10 & 2.50 & 15.7 & SPO \\
\hline & 0.010 & 0.009 & 0.02 & 0.002 & 0.3 & 0.2 & - & 0.06 & 0.2 & \\
\hline \multirow[t]{2}{*}{ SX788 } & 0.290 & 0.73 & 20.0 & 0.949 & 5.9 & 209.1 & 185.20 & 2.6 & 16.3 & SPO \\
\hline & 0.011 & 0.01 & 0.2 & 0.001 & 0.3 & 0.1 & - & 0.1 & 0.2 & \\
\hline \multirow[t]{2}{*}{ SX793 } & 0.005 & 0.997 & 113.99 & 0.749 & 430 & 119.6 & 185.27 & -0.4 & 59.59 & SPO \\
\hline & 0.008 & 0.006 & 0.09 & 0.001 & 770 & 0.2 & - & 0.5 & 0.09 & \\
\hline \multirow[t]{2}{*}{ SX798 } & 0.417 & 0.760 & 6.43 & 0.577 & 4.22 & 269.04 & 186.10 & 3.04 & 22.22 & SPO \\
\hline & 0.004 & 0.003 & 0.04 & 0.001 & 0.05 & 0.05 & - & 0.02 & 0.08 & \\
\hline \multirow[t]{2}{*}{ SX804 } & 0.137 & 0.863 & 69.28 & 1.001 & 13.6 & 178.0 & 186.18 & 1.1 & 40.99 & SPO \\
\hline & 0.006 & 0.006 & 0.09 & 0.001 & 0.6 & 0.2 & - & 0.1 & 0.08 & \\
\hline \multirow{2}{*}{ SX820 } & 0.390 & 0.758 & 2.71 & 0.621 & 4.51 & 82.63 & 27.69 & 2.94 & 21.04 & SPO \\
\hline & 0.002 & 0.001 & 0.01 & 0.001 & 0.03 & 0.03 & - & 0.01 & 0.04 & \\
\hline SX961 & 0.315 & 0.69 & 69.3 & 0.983 & 5.4 & 176.60 & 282.85 & 2.0 & 40.1 & QUA \\
\hline & 0.010 & 0.01 & 0.2 & 0.001 & 0.2 & 0.06 & - & 0.2 & 0.2 & \\
\hline SX962 & 0.318 & 0.69 & 71.0 & 0.978 & 5.3 & 170.5 & 282.85 & 2.0 & 40.9 & QUA \\
\hline & 0.017 & 0.02 & 0.3 & 0.001 & 0.3 & 0.2 & - & 0.3 & 0.3 & \\
\hline SX983 & 0.375 & 0.726 & 7.18 & 0.731 & 4.60 & 249.4 & 31.72 & 2.93 & 19.24 & SPO \\
\hline & 0.003 & 0.002 & 0.03 & 0.001 & 0.04 & 0.2 & - & 0.02 & 0.03 & \\
\hline SX988 & 0.308 & 0.868 & 10.1 & 0.430 & 6.1 & 283.4 & 31.89 & 2.37 & 29.1 & SPO \\
\hline & 0.015 & 0.007 & 0.2 & 0.003 & 0.3 & 0.3 & - & 0.07 & 0.2 & \\
\hline SX990 & 0.357 & 0.733 & 7.81 & 0.748 & 4.9 & 246.78 & 31.92 & 2.85 & 19.1 & SPO \\
\hline & 0.006 & 0.005 & 0.08 & 0.001 & 0.1 & 0.07 & - & 0.04 & 0.1 & \\
\hline SX1022 & 0.341 & 0.760 & 15.30 & 0.705 & 5.17 & 253.02 & 126.91 & 2.71 & 21.18 & SPO \\
\hline & 0.004 & 0.003 & 0.06 & 0.001 & 0.06 & 0.03 & - & 0.03 & 0.07 & \\
\hline SX1036 & 0.309 & 0.714 & 17.10 & 0.925 & 5.55 & 217.59 & 145.92 & 2.66 & 15.93 & SPO \\
\hline & 0.004 & 0.004 & 0.08 & 0.001 & 0.09 & 0.07 & - & 0.04 & 0.07 & \\
\hline SX1041 & 0.509 & 0.955 & 21.7 & 0.088 & 3.84 & 330.3 & 146.01 & 2.99 & 38.4 & SPO \\
\hline & 0.010 & 0.001 & 0.4 & 0.001 & 0.08 & 0.2 & - & 0.07 & 0.2 & \\
\hline SX1044 & 0.281 & 0.73 & 133.8 & 0.960 & 6.2 & 208.6 & 146.03 & 0.7 & 62.5 & SPO \\
\hline & 0.029 & 0.03 & 0.2 & 0.002 & 0.7 & 0.8 & - & 0.3 & 0.4 & \\
\hline SX1061 & 0.160 & 0.86 & 107 & 0.89 & 12 & 137.0 & 146.96 & 0 & 56.1 & SPO \\
\hline & 0.037 & 0.03 & 1 & 0.01 & 3 & 2.6 & - & 1 & 0.1 & \\
\hline SX1064 & 0.068 & 0.94 & 101.2 & 0.918 & 28 & 143.8 & 146.99 & 0.1 & 54.9 & SPO \\
\hline & 0.013 & 0.01 & 0.1 & 0.001 & 5 & 0.3 & - & 0.2 & 0.2 & \\
\hline SX1065 & 0.035 & 0.97 & 141.2 & 0.751 & 60 & 241 & 146.99 & -0.6 & 65.0 & SPO \\
\hline & 0.042 & 0.03 & 0.3 & 0.006 & 70 & 1 & - & 0.9 & 0.5 & \\
\hline SX1096 & 0.370 & 0.823 & 139.0 & 0.479 & 4.9 & 279.6 & 147.93 & 1.3 & 59.1 & SPO \\
\hline & 0.016 & 0.006 & 0.3 & 0.005 & 0.2 & 0.9 & - & 0.2 & 0.2 & \\
\hline SX1101 & 0.049 & 0.954 & 129.17 & 0.946 & 40 & 29.9 & 327.97 & -0.5 & 63.71 & SPO \\
\hline & 0.007 & 0.006 & 0.08 & 0.001 & 5 & 0.1 & - & 0.1 & 0.07 & \\
\hline SX1104 & 0.372 & 0.6 & 143 & 1.010 & 4 & 175 & 148.00 & 1 & 64 & SPO \\
\hline & 0.103 & 0.1 & 1 & 0.001 & 1 & 2 & - & 1 & 1 & \\
\hline SX1106 & 0.153 & 0.85 & 157.9 & 0.995 & 12 & 164.5 & 148.02 & -0.3 & 68.3 & SPO \\
\hline & 0.016 & 0.02 & 0.08 & 0.001 & 1 & 0.2 & - & 0.2 & 0.2 & \\
\hline SX1114 & 0.575 & 0.681 & 6.7 & 0.554 & 2.92 & 94.9 & 55.43 & 3.83 & 20.9 & SPO \\
\hline & 0.015 & 0.008 & 0.2 & 0.006 & 0.09 & 0.9 & - & 0.08 & 0.2 & \\
\hline SX1117 & 0.386 & 0.868 & 5.95 & 0.343 & 4.8 & 113.9 & 55.45 & 2.70 & 29.9 & SPO \\
\hline & 0.009 & 0.004 & 0.07 & 0.002 & 0.1 & 0.2 & - & 0.04 & 0.2 & \\
\hline SX1122 & 0.506 & 0.780 & 6.11 & 0.434 & 3.52 & 106.21 & 55.51 & 3.40 & 25.5 & TAU \\
\hline & 0.008 & 0.004 & 0.04 & 0.002 & 0.06 & 0.07 & - & 0.04 & 0.2 & \\
\hline SX1128 & 0.421 & 0.832 & 5.28 & 0.398 & 4.3 & 288.37 & 235.52 & 2.94 & 27.7 & TAU \\
\hline & 0.012 & 0.006 & 0.07 & 0.003 & 0.1 & 0.08 & - & 0.06 & 0.2 & \\
\hline SX1133 & 0.020 & 0.98 & 163.0 & 0.980 & 100 & 169.6 & 235.56 & -1 & 71.5 & LEO \\
\hline & 0.032 & 0.03 & 0.2 & 0.001 & 170 & 0.3 & - & 2 & 0.3 & \\
\hline SX1135 & 0.053 & 0.95 & 160.3 & 0.985 & 37 & 172.6 & 235.56 & -0.9 & 70.9 & LEO \\
\hline & 0.027 & 0.03 & 0.3 & 0.001 & 20 & 0.9 & - & 0.6 & 0.3 & \\
\hline SX1150 & 0.146 & 0.86 & 161.5 & 0.985 & 12.7 & 173.3 & 235.61 & -0.4 & 70.0 & LEO \\
\hline & 0.028 & 0.03 & 0.2 & 0.001 & 2.7 & 0.5 & - & 0.3 & 0.3 & \\
\hline
\end{tabular}


Table 5. continued.

\begin{tabular}{|c|c|c|c|c|c|c|c|c|c|c|}
\hline Spectrum & $\begin{array}{c}1 / a \\
(1 / \mathrm{AU})\end{array}$ & $e$ & $\begin{array}{c}i \\
\left({ }^{\circ}\right)\end{array}$ & $\begin{array}{c}q \\
(\mathrm{AU})\end{array}$ & $\begin{array}{c}Q \\
(\mathrm{AU})\end{array}$ & $\begin{array}{l}\omega \\
\left({ }^{\circ}\right)\end{array}$ & $\Omega$ & $\begin{array}{l}T_{j} \\
\left({ }^{\circ}\right)\end{array}$ & $\begin{array}{c}v_{\mathrm{g}} \\
\left(\mathrm{km} \mathrm{s}^{-1}\right)\end{array}$ & Shower \\
\hline \multirow{2}{*}{ SX1191 } & 0.381 & 0.889 & 14.53 & 0.291 & 4.96 & 300.4 & 16.81 & 2.61 & 32.87 & SPO \\
\hline & 0.003 & 0.001 & 0.06 & 0.001 & 0.03 & 0.1 & - & 0.02 & 0.03 & \\
\hline \multirow[t]{2}{*}{ SX1194 } & 0.395 & 0.867 & 3.1 & 0.335 & 4.7 & 295.6 & 16.91 & 2.75 & 30.6 & SPO \\
\hline & 0.011 & 0.003 & 0.2 & 0.003 & 0.1 & 0.4 & - & 0.05 & 0.2 & \\
\hline \multirow[t]{2}{*}{ SX1206 } & 0.373 & 0.758 & 7.12 & 0.650 & 4.71 & 259.3 & 17.83 & 2.87 & 21.69 & SPO \\
\hline & 0.004 & 0.003 & 0.04 & 0.001 & 0.06 & 0.1 & - & 0.02 & 0.07 & \\
\hline \multirow[t]{2}{*}{ SX1217 } & 0.482 & 0.952 & 21 & 0.099 & 4.1 & 327.9 & 19.01 & 2.9 & 39.2 & SPO \\
\hline & 0.023 & 0.003 & 1 & 0.004 & 0.2 & 0.8 & - & 0.2 & 0.3 & \\
\hline \multirow[t]{2}{*}{ SX1582 } & 0.087 & 0.92 & 80.7 & 0.922 & 40 & 209.6 & 32.45 & 0.4 & 46.9 & LYR \\
\hline & 0.012 & 0.01 & 0.2 & 0.001 & 20 & 0.3 & - & 0.3 & 0.2 & \\
\hline \multirow[t]{2}{*}{ SX1594 } & 0.113 & 0.985 & 13.0 & 0.134 & 17 & 318.4 & 45.04 & 1.03 & 41.1 & SPO \\
\hline & 0.010 & 0.001 & 0.3 & 0.001 & 2 & 0.1 & - & 0.04 & 0.2 & \\
\hline \multirow[t]{2}{*}{ SXDRA06 } & 0.304 & 0.697 & 31.5 & 0.996 & 5.6 & 173.23 & 195.05 & 2.6 & 20.6 & DRA \\
\hline & 0.007 & 0.007 & 0.1 & 0.001 & 0.2 & 0.05 & - & 0.1 & 0.1 & \\
\hline \multirow[t]{2}{*}{ SX1734 } & 0.018 & 0.983 & 79.48 & 0.923 & 110 & 213.57 & 31.97 & 0.31 & 46.96 & LYR \\
\hline & 0.003 & 0.003 & 0.03 & 0.001 & 20 & 0.05 & - & 0.04 & 0.04 & \\
\hline \multirow[t]{2}{*}{ SX1738 } & 0.023 & 0.979 & 80.4 & 0.914 & 90 & 215.3 & 32.05 & 0.3 & 47.3 & LYR \\
\hline & 0.008 & 0.007 & 0.1 & 0.001 & 30 & 0.2 & - & 0.1 & 0.1 & \\
\hline \multirow[t]{2}{*}{ SX1751 } & 0.010 & 0.991 & 79.06 & 0.915 & 200 & 215.0 & 33.09 & 0.28 & 46.86 & LYR \\
\hline & 0.005 & 0.004 & 0.06 & 0.001 & 100 & 0.1 & - & 0.09 & 0.06 & \\
\hline \multirow[t]{2}{*}{ SX1786 } & 0.013 & 0.988 & 113.6 & 0.954 & 157 & 151.9 & 139.46 & -0.4 & 59.96 & PER \\
\hline & 0.005 & 0.005 & 0.1 & 0.001 & 70 & 0.1 & - & 0.2 & 0.06 & \\
\hline \multirow[t]{2}{*}{ SX1798 } & -0.011 & 1.01 & 115.4 & 0.947 & -200 & 150.5 & 139.54 & -0.6 & 60.43 & PER \\
\hline & 0.007 & 0.01 & 0.1 & 0.001 & 100 & 0.2 & - & 0.3 & 0.08 & \\
\hline \multirow[t]{2}{*}{ SX1802 } & 0.039 & 0.96 & 115.1 & 0.960 & 50 & 153.3 & 139.56 & -0.3 & 59.9 & PER \\
\hline & 0.016 & 0.02 & 0.1 & 0.001 & 20 & 0.3 & - & 0.3 & 0.2 & \\
\hline
\end{tabular}

Table 6. Total intensities of lines $\mathrm{Mg}-2, \mathrm{Na}-1$ and $\mathrm{Fe}-15$.

\begin{tabular}{|c|c|c|c|c|c|c|c|}
\hline Spectrum & $\begin{array}{c}\mathrm{Mg}-2 \\
(\mathrm{~J} / \mathrm{sr})\end{array}$ & $\begin{array}{c}\mathrm{Na}-1 \\
(\mathrm{~J} / \mathrm{sr})\end{array}$ & $\begin{array}{c}\mathrm{Fe}-15 \\
(\mathrm{~J} / \mathrm{sr})\end{array}$ & spectrum & $\begin{array}{c}\mathrm{Mg}-2 \\
(\mathrm{~J} / \mathrm{sr})\end{array}$ & $\begin{array}{c}\mathrm{Na}-1 \\
(\mathrm{~J} / \mathrm{sr})\end{array}$ & $\begin{array}{c}\mathrm{Fe}-15 \\
(\mathrm{~J} / \mathrm{sr})\end{array}$ \\
\hline SX001 & $5.06 \times 10^{-1}$ & $1.56 \times 10^{-1}$ & $2.61 \times 10^{0}$ & SX785 & $7.36 \times 10^{-1}$ & $1.49 \times 10^{0}$ & $5.21 \times 10^{-1}$ \\
\hline SX002 & $1.72 \times 10^{0}$ & $1.09 \times 10^{0}$ & $9.57 \times 10^{-1}$ & SX788 & $2.08 \times 10^{0}$ & $4.25 \times 10^{0}$ & $1.78 \times 10^{0}$ \\
\hline SX008 & $2.34 \times 10^{0}$ & $3.86 \times 10^{-1}$ & $7.89 \times 10^{-1}$ & SX793 & $1.06 \times 10^{1}$ & $6.15 \times 10^{0}$ & $5.15 \times 10^{0}$ \\
\hline SX015 & $2.90 \times 10^{0}$ & $1.67 \times 10^{0}$ & $1.56 \times 10^{0}$ & SX798 & $2.96 \times 10^{0}$ & $5.09 \times 10^{0}$ & $2.12 \times 10^{0}$ \\
\hline SX045 & $1.80 \times 10^{0}$ & $6.43 \times 10^{-1}$ & $7.09 \times 10^{-1}$ & SX804 & $6.35 \times 10^{0}$ & $3.37 \times 10^{0}$ & $3.44 \times 10^{0}$ \\
\hline SX089 & $3.75 \times 10^{0}$ & $7.80 \times 10^{0}$ & $2.19 \times 10^{0}$ & SX820 & $3.54 \times 10^{1}$ & $9.76 \times 10^{1}$ & $2.22 \times 10^{1}$ \\
\hline SX090 & $2.62 \times 10^{0}$ & $2.59 \times 10^{0}$ & $1.64 \times 10^{0}$ & SX961 & $1.02 \times 10^{1}$ & $6.42 \times 10^{0}$ & $4.93 \times 10^{0}$ \\
\hline SX092 & $1.35 \times 10^{1}$ & $3.78 \times 10^{0}$ & $4.12 \times 10^{0}$ & SX962 & $2.05 \times 10^{0}$ & $6.39 \times 10^{-1}$ & $1.49 \times 10^{0}$ \\
\hline 116 & $2.32 \times 10^{1}$ & $3.55 \times 10^{1}$ & $9.45 \times 10^{0}$ & 983 & $7.51 \times 10^{0}$ & $1.72 \times 10^{1}$ & $3.24 \times 10^{0}$ \\
\hline 143 & $6.02 \times 10^{0}$ & $1.97 \times 10^{1}$ & $5.45 \times 10^{0}$ & 88 & $2.92 \times 10^{0}$ & $1.37 \times 10^{0}$ & $1.89 \times 10^{0}$ \\
\hline 150 & $4.40 \times 10^{-1}$ & $3.36 \times 10^{0}$ & $3.79 \times 10^{-1}$ & 90 & $1.26 \times 10^{0}$ & $1.37 \times 10^{0}$ & $1.40 \times 10^{0}$ \\
\hline 151 & $2.97 \times 10^{0}$ & $1.99 \times 10^{-1}$ & $1.94 \times 10^{0}$ & SX1022 & $5.28 \times 10^{0}$ & $8.38 \times 10^{0}$ & $3.99 \times 10^{0}$ \\
\hline 158 & $1.06 \times 10^{0}$ & $4.85 \times 10^{-1}$ & $7.40 \times 10^{-1}$ & SX1036 & $6.03 \times 10^{-1}$ & $9.26 \times 10^{-1}$ & $5.40 \times 10^{-1}$ \\
\hline SX211 & $3.57 \times 10^{1}$ & $2.05 \times 10^{1}$ & $1.05 \times 10^{1}$ & SX & $4.36 \times 10^{0}$ & $1.67 \times 10^{0}$ & $4.70 \times 10^{0}$ \\
\hline $\mathrm{S}$ & $4.72 \times 10^{0}$ & $2.26 \times 10^{0}$ & $1.71 \times 10^{0}$ & 44 & $2.88 \times 10^{-1}$ & $2.10 \times 10^{-2}$ & $6.00 \times 10^{-2}$ \\
\hline $\mathrm{S}$ & $4.26 \times 10^{0}$ & $2.88 \times 10^{0}$ & $1.87 \times 10^{0}$ & 61 & $3.31 \times 10^{0}$ & $1.76 \times 10^{0}$ & $1.81 \times 10^{0}$ \\
\hline $\mathrm{S}$ & $2.32 \times 10^{1}$ & $3.38 \times 10^{1}$ & $9.32 \times 10^{0}$ & 4 & $5.83 \times 10^{0}$ & $4.48 \times 10^{0}$ & $2.07 \times 10^{0}$ \\
\hline $\mathrm{S}$ & $1.50 \times 10^{1}$ & $2.55 \times 10^{0}$ & $7.03 \times 10^{0}$ & & $3.67 \times 10^{-1}$ & $2.21 \times 10^{-1}$ & $2.13 \times 10^{-1}$ \\
\hline & $2.91 \times 10^{1}$ & $1.58 \times 10^{1}$ & $1.09 \times 10^{1}$ & & $2.85 \times 10^{-1}$ & $7.10 \times 10^{-2}$ & $1.40 \times 10^{-1}$ \\
\hline & $5.20 \times 10^{0}$ & $5.76 \times 10^{-1}$ & $2.42 \times 10^{0}$ & & $1.19 \times 10^{0}$ & $1.50 \times 10^{0}$ & $7.56 \times 10^{-1}$ \\
\hline & $4.04 \times 10^{0}$ & $4.31 \times 10^{-1}$ & $6.40 \times 10^{-1}$ & & $4.55 \times 10^{-1}$ & $1.80 \times 10^{-2}$ & $1.14 \times 10^{-1}$ \\
\hline & $3.10 \times 10^{-2}$ & $2.00 \times 10^{-3}$ & $3.86 \times 10^{-1}$ & & $7.81 \times 10^{-1}$ & $3.31 \times 10^{-1}$ & $5.07 \times 10^{-1}$ \\
\hline & $2.08 \times 10^{0}$ & $3.95 \times 10^{-1}$ & $1.07 \times 10^{0}$ & & $1.39 \times 10^{-1}$ & $9.50 \times 10^{-2}$ & $1.20 \times 10^{0}$ \\
\hline & $1.87 \times 10^{0}$ & $4.89 \times 10^{-1}$ & $6.58 \times 10^{-1}$ & & $3.41 \times 10^{0}$ & $7.07 \times 10^{-1}$ & $2.35 \times 10^{0}$ \\
\hline & $1.12 \times 10^{0}$ & $6.89 \times 10^{-1}$ & $6.23 \times 10^{-1}$ & & $8.07 \times 10^{0}$ & $1.05 \times 10^{1}$ & $8.46 \times 10^{0}$ \\
\hline & $5 \times 10$ & $4.68 \times 10^{-1}$ & $4.55 \times 10^{-1}$ & & $6.75 \times 10^{-1}$ & $5.08 \times 10^{-1}$ & $5.86 \times 10^{-1}$ \\
\hline & $3.50 \times 10^{-1}$ & $1.75 \times 10^{0}$ & $4.30 \times 10^{-1}$ & & $1.47 \times 10^{0}$ & $1.02 \times 10^{0}$ & $1.30 \times 10^{0}$ \\
\hline & $3.92 \times 10^{0}$ & $6.77 \times 10^{0}$ & $3.11 \times 10^{0}$ & & $4.35 \times 10^{-1}$ & $2.10 \times 10^{-1}$ & $4.43 \times 10^{-1}$ \\
\hline & $5.22 \times 10^{0}$ & $8.39 \times 10^{0}$ & $3.86 \times 10^{0}$ & & $1.46 \times 10^{0}$ & $9.12 \times 10^{-1}$ & $1.93 \times 10^{0}$ \\
\hline & $1.13 \times 10^{-1}$ & $1.00 \times 10^{-2}$ & $8.78 \times 10^{-1}$ & & $1.69 \times 10^{0}$ & $6.04 \times 10^{-1}$ & $6.24 \times 10^{-1}$ \\
\hline & $4.00 \times 10^{-2}$ & $6.00 \times 10^{-3}$ & $3.89 \times 10^{-1}$ & & $3.55 \times 10^{-1}$ & $5.00 \times 10^{-2}$ & $3.33 \times 10^{0}$ \\
\hline SX696 & $7.31 \times 10^{0}$ & $4.54 \times 10^{-1}$ & $7.46 \times 10^{-1}$ & SX1206 & $3.51 \times 10^{0}$ & $4.68 \times 10^{0}$ & $2.82 \times 10^{0}$ \\
\hline
\end{tabular}


V. Vojáček et al.: Catalogue of representative meteor spectra

Table 6. continued.

\begin{tabular}{lccc|cccc}
\hline \hline Spectrum & $\begin{array}{c}\mathrm{Mg}-2 \\
(\mathrm{~J} / \mathrm{sr})\end{array}$ & $\begin{array}{c}\mathrm{Na}-1 \\
(\mathrm{~J} / \mathrm{sr})\end{array}$ & $\begin{array}{c}\mathrm{Fe}-15 \\
(\mathrm{~J} / \mathrm{sr})\end{array}$ & spectrum & $\begin{array}{c}\mathrm{Mg}-2 \\
(\mathrm{~J} / \mathrm{sr})\end{array}$ & $\begin{array}{c}\mathrm{Na}-1 \\
(\mathrm{~J} / \mathrm{sr})\end{array}$ & $\begin{array}{c}\mathrm{Fe}-15 \\
(\mathrm{~J} / \mathrm{sr})\end{array}$ \\
\hline SX700 & $5.40 \times 10^{0}$ & $6.42 \times 10^{-1}$ & $2.27 \times 10^{0}$ & SX1217 & $9.40 \times 10^{-1}$ & $2.90 \times 10^{-2}$ & $3.46 \times 10^{-1}$ \\
SX708 & $6.97 \times 10^{0}$ & $6.43 \times 10^{0}$ & $4.27 \times 10^{0}$ & SX1582 & $6.75 \times 10^{0}$ & $5.07 \times 10^{0}$ & $3.30 \times 10^{0}$ \\
SX709 & $3.21 \times 10^{-1}$ & $2.43 \times 10^{-1}$ & $2.57 \times 10^{-1}$ & SX1594 & $1.24 \times 10^{0}$ & $3.53 \times 10^{-1}$ & $1.11 \times 10^{0}$ \\
SX713 & $3.29 \times 10^{-1}$ & $9.54 \times 10^{-1}$ & $3.68 \times 10^{-1}$ & SXDRA06 & $2.54 \times 10^{0}$ & $4.72 \times 10^{0}$ & $3.28 \times 10^{0}$ \\
SX718 & $4.35 \times 10^{-1}$ & $5.64 \times 10^{-1}$ & $4.04 \times 10^{-1}$ & SX1734 & $1.16 \times 10^{0}$ & $6.88 \times 10^{-1}$ & $1.03 \times 10^{0}$ \\
SX719 & $7.15 \times 10^{-1}$ & $7.68 \times 10^{-1}$ & $6.77 \times 10^{-1}$ & SX1738 & $1.54 \times 10^{0}$ & $5.46 \times 10^{-1}$ & $7.71 \times 10^{-1}$ \\
SX726 & $3.21 \times 10^{0}$ & $3.83 \times 10^{0}$ & $3.16 \times 10^{0}$ & SX1751 & $1.41 \times 10^{0}$ & $5.58 \times 10^{-1}$ & $4.56 \times 10^{-1}$ \\
SX731 & $1.37 \times 10^{0}$ & $1.38 \times 10^{-1}$ & $4.67 \times 10^{-1}$ & SX1786 & $3.28 \times 10^{0}$ & $2.33 \times 10^{0}$ & $2.72 \times 10^{0}$ \\
SX738 & $3.15 \times 10^{0}$ & $2.89 \times 10^{-1}$ & $1.49 \times 10^{0}$ & SX1798 & $3.80 \times 10^{0}$ & $2.28 \times 10^{0}$ & $1.74 \times 10^{0}$ \\
SX784 & $1.92 \times 10^{0}$ & $7.61 \times 10^{-1}$ & $6.38 \times 10^{-1}$ & SX1802 & $6.60 \times 10^{0}$ & $5.11 \times 10^{0}$ & $2.84 \times 10^{0}$ \\
\hline
\end{tabular}

